\title{
一锅法合成多取代色酮并双环吡啶类化合物
}

\author{
陈亮王保取赵宇澄严胜骄* 林 军*
}

(云南大学化学科学与工程学院 教育部自然资源药物化学重点实验室 昆明 650091)

\begin{abstract}
摘要 建立了一种简捷、绿色合成多取代色酮并双环吡啶类化合物的合成方法. 该方法是利用色酮-3-甲酫 1 与 $N$-芐基 硝基烯酮缩胺(NBNKAs，2)在乙醇中反应，一步合成色酮并双环吡啶化合物. 通过直接抽滤得目标产物 $\mathbf{3}$, 无需额外的 后处理操作. 该方法具有合成成本低, 反应溶剂生物相容性好, 反应条件温和，原子经济性，高产率 $(93 \sim 98 \%)$ 及产物 具有潜在的生物活性等显著优势.
\end{abstract}

关键词 环境友好; 原子经济; 无催化剂; 咪唑并吡啶; 新烟碱类

\section{One-Pot Synthesis of Multisubstituted Chromone-Fused Bicyclic Pyridine Compounds}

\author{
Chen, Liang Wang, Baoqu Zhao, Yucheng Yan, Shengjiao* Lin, Jun* \\ (Key Laboratory of Medicinal Chemistry for Natural Resource, Ministry of Education, School of Chemical Science and \\ Technology, Yunnan University, Kunming 650091)
}

\begin{abstract}
A concise and environment friendly route for the synthesis of multisubstituted chromone-fused bicyclic pyridine compounds via one-step reaction of chromone-3-carboxaldehyde $\mathbf{1}$ and $N$-benzyl nitro ketene aminals (NBNKAs, 2) in ethanol media has been developed. The targeted compounds $\mathbf{3}$ can efficiently obtain by filter without extra post-treatment. The reaction is particularly attractive due to following features: low-cost and biocompatibility solvent, mild temperature, atom economy, high yields, and potential biological activity.

Keywords environment friendly; atom economy; catalyst-free; bicyclic pyridine; neonicotinoids
\end{abstract}

化工产品和药物的生产离不开化学合成. 化学合成 丰富人们物质世界的同时，不可避免地带来很多问题. 包括资源和能源的浪费, 以及环境污染等 ${ }^{[1,2]}$. 而如何应 对由化学合成带来的污染已成为现代化学工作者急需 思考和面对的问题. 理想的化学合成应该是一个绿色环 保和高效低碳的过程 ${ }^{[3,4]}$, 美国著名化学家 $\operatorname{Trost}^{[5]}$ 在 1991 年提出了 “原子经济性” 的原则, 认为在设计合成 路线的时候应该尽可能地消除或者减少有害物质的产 生, 美国环境保护组织(U.S. EPA)也倡导化学反应应满 足 “绿色化学” 的要求 ${ }^{[6]}$. 而本文旨在探索如何绿色、 高效构筑 C-C 和 C $-\mathrm{N}$ 键并合成含色酮骨架结构的双 环咪唑并吡啶类化合物的. 含氮杂环化合物在合成药 物 $^{[7,8]}$ 及天然产物中扮演着十分重要的角色 ${ }^{[9,10]}$.

含氮杂环化合物具有丰富多样的生物活性，比如说 抗癌 ${ }^{[11]}$ 、抗病毒 ${ }^{[12]}$ 、治疗心脑血管疾病 $\left.{ }^{[13}\right]$ 、镇静安眠 等 ${ }^{[14,15]}$. 咪唑并吡啶作为含氮杂环化合物家族中较为重 要的一员, 已经在很多临床药物中发挥着重要的作 用 ${ }^{[16,17]}$, 已经商品化的药物包括奥普力农 ${ }^{[18]}$ 、法倔 唑 ${ }^{[19]}$ 、唑吡旦 ${ }^{[20]}$ 等.

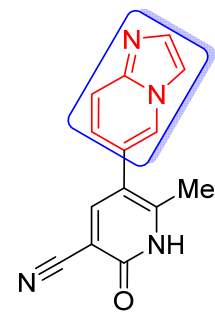

奥普力农 (强心药)

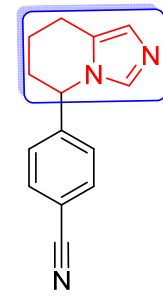

法倔唑

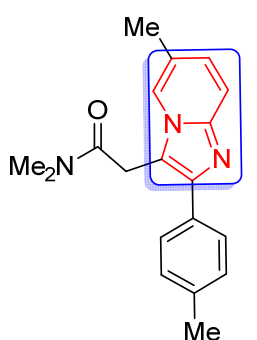

唑吡旦(安眠药)

\footnotetext{
* Corresponding authors. E-mail: yansj@ynu.edu.cn; linjun@ynu.edu.cn

Received December 12, 2016; revised January 21, 2017; published online February 20, 2017.

Project supported by the National Natural Science Foundation of China (Nos. U1202221, 21362042, 21262042, 21662042), the Talent Found in Yunnan Province (No. 2012HB001), and the Excellent Young Talents of Yunnan University (No. XT412003).

国家自然科学基金(Nos. U1202221, 21362042, 21262042, 21662042)、云南省后备人才(No. 2012HB001)、云南大学青年英才计划(No. XT412003)资助 项目.
} 
新烟碱型类似物 (如吡虫啉 ${ }^{[21]} 、 6-\mathrm{Cl}-\mathrm{PMNI}^{[22] 、}$ 哌虫 啶 ${ }^{[23]}$ 等)因其良好的杀虫活性以及较低的哺乳动物毒性, 迅速发展并成为继有机磷农药、氨基甲酸盐及除虫菊酯 之后的第四代新型农药 ${ }^{[24,25]}$. 而新烟碱类似物, 因其特 殊的结构特征: (1)柔性的苄基侧链, (2)强吸电子的硝基, (3)芳香核，(4)和双键相连的胺基. 李忠课题组 ${ }^{[26,27]}$ 开展 一系列新烟碱类似物的研究工作, 合成获得很多活性化 合物. 其中, 新烟碱类似物 $N$-苄基硝基烯酮缩胺(NBNKAs，2)属于多功能合成砌块即杂环烯酮缩胺(HKAs)类 化合物 ${ }^{[28,29]}$. NBNKAs 在构建具有潜在生物活性的含氮 杂环化合物方面有独特的优势 ${ }^{[30,31]}$.<smiles>O=[N+]([O-])/N=C1\NCCN1Cc1ccc(Cl)nc1</smiles>

吡虫啉<smiles>CC1CC(Oc2ccc(CN3CCNC3=C[N+](=O)[O-])cn2)N2CCN(Cc3ccc(Cl)nc3)C2=C1[N+](=O)[O-]</smiles>

黄酮类药物在抗癌、抗衰老及治疗血管疾病方面发 挥着重要作用 ${ }^{[32,33]}$. 黄酮类化合物均含有色酮母核. 本 文拟根据药物拼合原理, 把色酮结构及新烟碱结构拼合 到目标化合物双环咪唑并吡啶类化合物中, 希望新杂合 物具有更优、更广谱的生物活性, 为今后进行生物活性 普笁打下基础.

本文目标产物的合成用取代色酮-3-甲醛化合物 1 与 2 反应, 通过 $2 \alpha-\mathrm{C}$ 选择性对色酮羰基加成脱水、氨 基对色酮双键进行迈克尔加成得目标产物(Eq. 1). 相对 其他文献报道的杂环构筑方法 ${ }^{[34]}$, 本方法符合绿色合 成原则, 操作简单, 反应条件温和, 反应时间短, 原子 利用率高, 产率高.

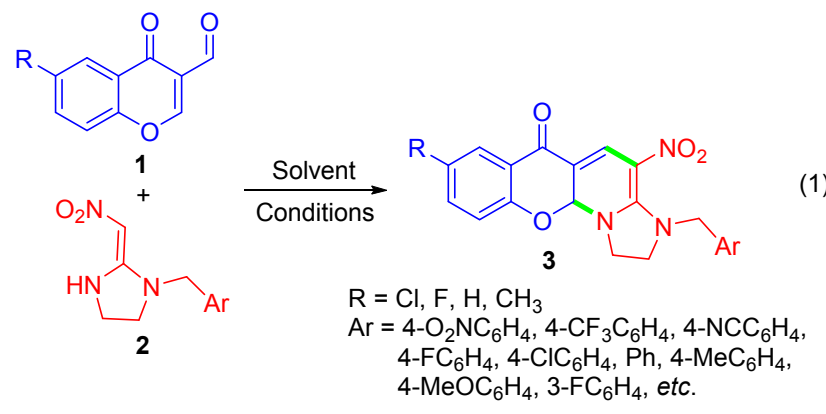

\section{1 结果与讨论}

\section{1 反应条件的篮选}

称取 $1.1 \mathrm{mmol}$ 的色酮-3-甲醛 $1 \mathbf{a}$ 和 $1.0 \mathrm{mmol}$ 的 1-(4硝基苄基)-2-(硝基亚甲基)咪唑烷(2a)加入到 $25 \mathrm{~mL}$ 圆底 烧瓶中, 加入 $15 \mathrm{~mL}$ 溶剂搅拌, 之后对反应溶剂、催化 剂、时间以及温度对产率的影响进行了篮选, 结果见表
1.

表 1 优化反应条件

Table 1 Optimization of reaction conditions ${ }^{a}$

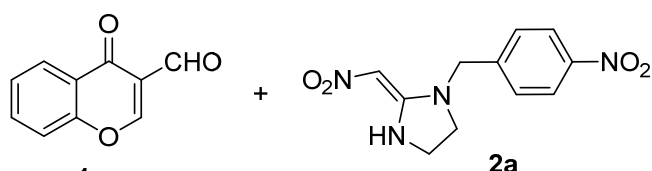

1a

$2 a$

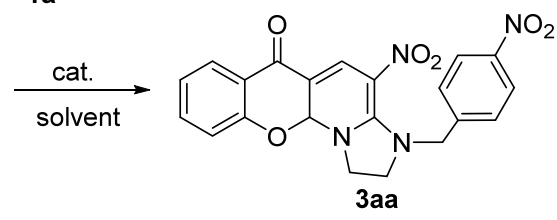

\begin{tabular}{|c|c|c|c|c|c|}
\hline Entry & Solvent & Catalyst & $T /{ }^{\circ} \mathrm{C}$ & Time/min & Yield $^{b} / \%$ \\
\hline 1 & $\mathrm{EtOH}$ & - & r.t. & 60 & 75 \\
\hline 2 & DCM & - & r.t. & 120 & 76 \\
\hline 3 & EtOH & - & Reflux & 20 & 96 \\
\hline 4 & DMF & - & Reflux & 30 & 72 \\
\hline 5 & Toluene & - & Reflux & 60 & 81 \\
\hline 6 & 1,4-Dioxane & - & Reflux & 60 & 84 \\
\hline 7 & $\mathrm{CH}_{3} \mathrm{CN}$ & 一 & Reflux & 60 & 74 \\
\hline 8 & $\mathrm{MeOH}$ & - & Reflux & 30 & 78 \\
\hline 9 & $\mathrm{H}_{2} \mathrm{O}$ & - & Reflux & 60 & 65 \\
\hline 10 & $\mathrm{EtOH}$ & $\mathrm{K}_{2} \mathrm{CO}_{3}$ & Reflux & 20 & 81 \\
\hline 11 & $\mathrm{EtOH}$ & $\mathrm{Et}_{3} \mathrm{~N}$ & Reflux & 20 & 90 \\
\hline 12 & $\mathrm{EtOH}$ & Piperidine & Reflux & 20 & 92 \\
\hline 13 & $\mathrm{EtOH}$ & $\mathrm{Cs}_{2} \mathrm{CO}_{3}$ & Reflux & 20 & 80 \\
\hline
\end{tabular}

${ }^{a}$ Reactions were carried out using $1 \mathbf{a}(1.1 \mathrm{mmol}), \mathbf{2 a}(1.0 \mathrm{mmol})$, and solvent $(15 \mathrm{~mL}) .{ }^{b}$ Isolated yield based on NBNKA $\mathbf{2 a}$.

最初我们尝试了在室温下, 分别以 $\mathrm{EtOH}$ 和 $N, N$-二 甲基甲酰胺(DMF)为溶剂，无催化剂条件下的反应，以 薄层色谱(TLC)作为检测手段，结果显示(表 1, Entries 1 2), 两种情况下都有目标化合物 $3 \mathbf{a a}$ 生成, 但是两种 条件下，原料都没有反应完. 然后又考查了 $\mathrm{EtOH}$ 作为 溶剂回流条件下的反应(表 1, Entry 3), 结果显示升高温 度对反应是有利的, 能在较短时间 (20 min) 以较高产率 (96\%)获得目标化合物 3aa. 我们进一步考察了不同溶 剂对反应的影响, 结果显示(表 1, Entries 4 9), 不论是 质子性溶剂 $\left(\mathrm{EtOH}, \mathrm{MeOH}, \mathrm{H}_{2} \mathrm{O}\right)$, 还是非质子性溶剂 (DCM, toluene, 1,4-dioxane)都能得到目标产物. 但是产 率存在一定的差异 $(72 \% \sim 65 \%)$, 我们推测可能是由于 目标产物最后都是以沉淀的形式从反应液中析出，所以 溶剂的极性对产率会造成影响，对产物溶解性好的溶剂 会使得产率下降，但这并不意味着对产物溶解性差的溶 剂就能给出高的产率(Entries 5,9). 部分溶剂由于没办 法对底物进行有效地溶解, 使得底物无法充分接触, 反 应受阻，导致反应底物和产物混在一起，影响后处理. 在最优溶剂选择为 $\mathrm{EtOH}$ 的基础上, 进一步研究了催化 剂对本反应的影响, 结果显示(表 1, Entries 10 13), 催 
化剂的加入反而降低了产率.

综合来看, 最优的合成条件为以乙醇作为溶剂, 底 物 $1.1: 1$, 无催化剂回流 $20 \mathrm{~min}$, 能够以优异的产率 (96\%)得目标化合物 3aa. 且反应结束后待反应液冷却 到室温，过滤后取沉淀干燥即可得纯的目标产物.

\section{2 目标产物及产率}

在最佳条件基础上, 为进一步的验证反应的普适 性，我们以色酮-3-甲醛 $\mathbf{1 a} \sim \mathbf{1 d}$ 与 $N$-苠基的硝基烯酮缩 胺 $\mathbf{2 a} \sim \mathbf{2 k}$ 反应, 成功合成 30 个双环味坐并吡啶类化合 物 3aa $3 \mathbf{d k}$. 结果详见表 2 .

实验过程中我们发现, 当色酮-3-甲醛的 C(6)位上 是吸电子基团(比如 $\mathrm{F}$ 和 $\mathrm{Cl}$ )时，反应的速度明显比 $\mathrm{C}(6)$
位上是给电子基团 $\mathrm{CH}_{3}$ 的时候快，根据我们推测的反应 机理(Scheme 1)来说, 有可能是色酮的苯环上有吸电子 基团的时候，能够提高色酮羰基碳的电正性，有利于 $N$ 苄基的硝基烯酮缩胺 $\alpha$-C 对其进攻，所以造成反应速度 加快. 根据以上推测, 实际的反应速率顺序应为: $\mathrm{F}>$ $\mathrm{Cl}>\mathrm{H}>\mathrm{CH}_{3}$, 但是实际实验观察结果则是 $\mathrm{Cl}>\mathrm{F}>\mathrm{H}>$ $\mathrm{CH}_{3}$. 造成上述结果的原因可能是由 $\mathrm{C}(6)$ 位是 $\mathrm{F}$ 的时候, $\mathrm{F}$ 原子容易和 $\mathrm{NH}$ 里的 $\mathrm{H}$ 原子形成氢键, 干扰了 $\mathrm{NH}$ 中 $\mathrm{H}$ 原子与色酮羰基上氧原子氢键的形成. 最终导致含 $\mathrm{F}$ 色酮的反应速度低于含 $\mathrm{Cl}$ 色酮.

通过的实验数据的分析可以发现，不论是色酮-3甲醛上取代基的种类还是 $N$-苄基硝基烯酮缩胺的芳香

表 2 一锅法合成化合物 $\mathbf{3}^{a}$

Table 2 One-pot protocol for the synthesis of compound 3<smiles>[R]c1ccc2occ(C=O)c(=O)c2c1</smiles><smiles>O=[N+]([O-])C=C1NCCN1CBr</smiles>

2<smiles>[R]c1ccc2c(c1)C(=O)C1=CC([N+](=O)[O-])=C3N(C[Te])CCN3C1O2</smiles>

3

\begin{tabular}{|c|c|c|c|c|c|c|}
\hline Entry & $\mathrm{R}$ & 1 & $\mathrm{Ar}$ & 2 & 3 & Yield $/ \%$ \\
\hline 1 & $\mathrm{H}$ & 1a & 4-Nitrophenyl & $2 a$ & 3aa & 96 \\
\hline 2 & $\mathrm{H}$ & 1a & 4-(Trifluoromethyl)phenyl & $2 b$ & 3ab & 98 \\
\hline 3 & $\mathrm{H}$ & 1a & 4-Cyanophenyl & $2 c$ & $3 a c$ & 93 \\
\hline 4 & $\mathrm{H}$ & 1a & 4-Fluorophenyl & 2d & 3ad & 96 \\
\hline 5 & $\mathrm{H}$ & $1 \mathbf{a}$ & 4-Chlorophenyl & $2 e$ & 3ae & 97 \\
\hline 6 & $\mathrm{H}$ & 1a & Phenyl & $2 f$ & 3af & 95 \\
\hline 7 & $\mathrm{H}$ & 1a & 4-Methylphenyl & $2 \mathrm{~g}$ & $3 a g$ & 97 \\
\hline 8 & $\mathrm{H}$ & 1a & 4-Methoxyphenyl & $2 \mathrm{~h}$ & 3ah & 96 \\
\hline 9 & $\mathrm{H}$ & 1a & 3-Fluorophenyl & $2 \mathbf{i}$ & 3ai & 94 \\
\hline 10 & $\mathrm{H}$ & 1a & 3,5-Difluorophenyl & $2 \mathbf{j}$ & 3aj & 94 \\
\hline 11 & $\mathrm{H}$ & 1a & 6-Chloropyridin-3-yl & $2 k$ & 3ak & 95 \\
\hline 12 & $\mathrm{H}$ & 1a & 2-Chlorothiazol-5-yl & 21 & 3al & 93 \\
\hline 13 & $\mathrm{~F}$ & $1 b$ & 4-(Trifluoromethyl)phenyl & $2 \mathbf{b}$ & $3 \mathbf{b b}$ & 95 \\
\hline 14 & $\mathrm{~F}$ & $1 b$ & 4-Cyanophenyl & $2 c$ & $3 \mathrm{bc}$ & 94 \\
\hline 15 & $\mathrm{~F}$ & $1 b$ & 4-Fluorophenyl & 2d & 3bd & 95 \\
\hline 16 & $\mathrm{~F}$ & $1 b$ & 4-Chlorophenyl & $2 e$ & 3be & 96 \\
\hline 17 & $\mathrm{~F}$ & $1 b$ & Phenyl & $2 f$ & $3 \mathrm{bf}$ & 97 \\
\hline 18 & $\mathrm{~F}$ & $1 \mathrm{~b}$ & 4-Methylphenyl & $2 \mathrm{~g}$ & $3 b g$ & 96 \\
\hline 19 & $\mathrm{~F}$ & $1 \mathrm{~b}$ & 4-Methoxyphenyl & $2 \mathrm{~h}$ & $3 b h$ & 95 \\
\hline 20 & $\mathrm{~F}$ & $1 b$ & 3-Fluorophenyl & $2 \mathbf{i}$ & $3 \mathbf{b i}$ & 94 \\
\hline 21 & $\mathrm{~F}$ & $1 b$ & 3,5-Difluorophenyl & $2 \mathbf{j}$ & $3 \mathbf{b j}$ & 98 \\
\hline 22 & $\mathrm{~F}$ & $1 b$ & 6-Chloropyridin-3-yl & $2 k$ & $3 b k$ & 96 \\
\hline 23 & $\mathrm{~F}$ & $1 b$ & 2-Chlorothiazol-5-yl & 21 & $3 \mathrm{bl}$ & 95 \\
\hline 24 & $\mathrm{Cl}$ & $1 \mathrm{c}$ & 4-(Trifluoromethyl)phenyl & $2 \mathbf{b}$ & $3 \mathrm{cb}$ & 95 \\
\hline 25 & $\mathrm{Cl}$ & $1 \mathrm{c}$ & 4-Fluorophenyl & 2d & $3 \mathrm{~cd}$ & 96 \\
\hline 26 & $\mathrm{Cl}$ & $1 \mathrm{c}$ & 4-Methylphenyl & $2 \mathrm{~g}$ & $3 \mathrm{cg}$ & 97 \\
\hline 27 & $\mathrm{Cl}$ & $1 \mathrm{c}$ & 4-Methoxyphenyl & $2 \mathrm{~h}$ & $3 \mathrm{ch}$ & 96 \\
\hline 28 & $\mathrm{CH}_{3}$ & 1d & 4-Cyanophenyl & $2 c$ & $3 d c$ & 98 \\
\hline 29 & $\mathrm{CH}_{3}$ & 1d & 4-Methoxyphenyl & $2 \mathrm{~h}$ & $3 d h$ & 98 \\
\hline 30 & $\mathrm{CH}_{3}$ & 1d & 6-Chloropyridin-3-yl & $2 \mathrm{k}$ & $3 \mathrm{dk}$ & 97 \\
\hline
\end{tabular}

${ }^{a}$ All reactions were run under the following conditions: 1 (1.1 mmol) and $\mathbf{2}(1.0 \mathrm{mmol})$ were refluxed in the solvent EtOH $(15 \mathrm{~mL})$ for 20 min. ${ }^{b}$ Isolated yield based on NBNKAs 2 


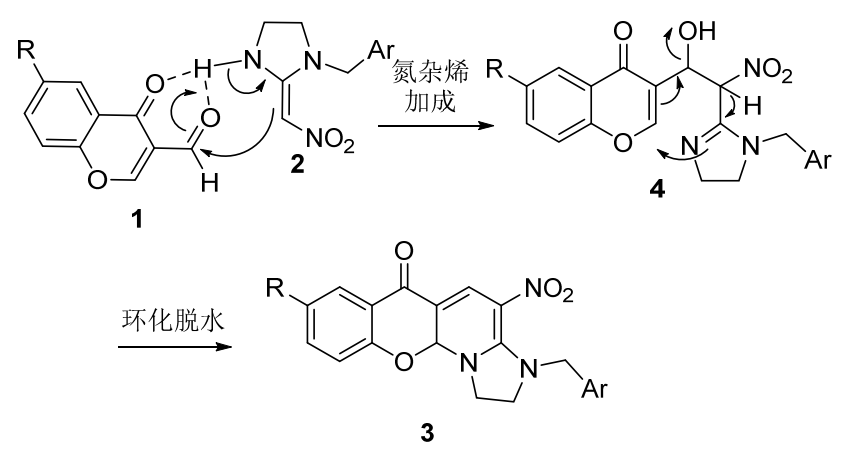

图式 1 合成目标产物 3 反应机理的推断

Scheme 1 Mechanism hypotheses for the synthesis of target compounds 3

环种类, 对反应的产率均无明显影响. 结合最终产物以 沉淀形式析出这一现象, 说明此反应有很好的热力学优 势以及较为广泛的适用性.

\section{3 反应机理}

我们推测反应机理如 Scheme 1 所示. NBNKAs 化合 物氨基上的 $\mathrm{H}$ 原子首先和色酮-3-甲醛羰基氧原子形成 分子间的氢键 ${ }^{[35,36]}$. 而氢键的形成提高了色酮-3-甲醛 中羰基碳原子的电正性, 使得其亲电反应活性提高. 另 一方面，分子间氢键的形成起到了定位作用，有利于 NBNKAs 中双键 $\alpha-C$ 对色酩醛基亲核加成得中间体 4 . 然后中间体 4 脱去一分子水, 而 $\mathrm{N}$ 进攻色酮的 $\mathrm{C}(2)$ 位点, 经过双键位置的重排，得到目标产物 3.

\section{2 结论}

本文建立了一种绿色、高效一步法合成多取代色酩 并双环吡定类衍生物的方法. 该方法利用 $\mathrm{C}(6)$ 位不同取 代基的色酮-3-甲醛与不同芳香取代的 $N$-茮基硝基烯酮 缩胺类化合物(NBNKAs) 反应快速合成一系列含色酮骨 架结构的咪唑并吡啶类化合物. 且反应结束后, 经过简 单过滤就能得到目标产物. 反应过程符合绿色化学的理 念, 溶剂环保, 整个反应只脱出一分子水, 反应原子利 用率高, 产率高. 另一方面, 合成所用两个原料本身就 具有很好的生物活性, 这为后续对产物的活性篮选提供 了良好的基础.

\section{3 实验部分}

\section{1 仪器和试剂}

IR: 傅里叶红外光谱仪(Thermo Nicolet Avatar 360 型); HRMS: 高分辨质谱仪 (Agilent CL/Msd TOF); NMR: 核磁共振仪 Bruck DRX500 $\left({ }^{1} \mathrm{H}: 500 \mathrm{MHz},{ }^{13} \mathrm{C}\right.$ : $125 \mathrm{MHz})$, Bruck DRX600 ( $\left.{ }^{1} \mathrm{H}: 600 \mathrm{MHz},{ }^{13} \mathrm{C}: 150 \mathrm{MHz}\right)$; 熔点仪(XT-4A 控温型显微熔点测定仪); 控温型电磁搅
拌器. $\mathrm{GF}_{254}$ 高效薄层层析板, 分析纯或化学纯试剂, NBNKAs 2 按文献[37]制备.

\section{2 化合物 3 的合成}

称取 $1.1 \mathrm{mmol}$ 色酮-3-甲醛 1 和 $1.0 \mathrm{mmol} \mathrm{N}$-芐基硝 基烯酮缩胺 2 共同加入到 $25 \mathrm{~mL}$ 圆底烧瓶中, 加入 15 $\mathrm{mL}$ 乙醇, 充分摚拌并加热回流 $20 \mathrm{~min}, \mathrm{TLC}$ 监测直至原 料消失，停止反应. 待反应液冷却至室温后过滤，取沉 淀在红外灯下干燥, 得到目标产物 $\mathbf{3 a a} \sim \mathbf{3 d k}$, 产率为 93\% 98\%.

4-硝基-3-(4-硝基苄基)-1,2,3,11a-四氢- $6 \mathrm{H}$-苯并吡喃 并[3,2-e]咪唑并[1,2-a]吡啶-6-酮(3aa): 黄色固体, m.p. $258 \sim 259{ }^{\circ} \mathrm{C} ;{ }^{1} \mathrm{H}$ NMR $\left(500 \mathrm{MHz}\right.$, DMSO- $\left.d_{6}\right) \delta: 8.25(\mathrm{~d}$, $J=9.0 \mathrm{~Hz}, 2 \mathrm{H}, \mathrm{ArH}), 7.92(\mathrm{~s}, 1 \mathrm{H}, \mathrm{CH}), 7.83 \sim 7.85(\mathrm{~m}$, $1 \mathrm{H}, \operatorname{ArH}), 7.68(\mathrm{~d}, J=9.0 \mathrm{~Hz}, 2 \mathrm{H}, \operatorname{ArH}), 7.61 \sim 7.65(\mathrm{~m}$, $1 \mathrm{H}, \operatorname{ArH}), 7.17 \sim 7.20(\mathrm{~m}, 1 \mathrm{H}, \operatorname{ArH}), 7.11(\mathrm{~d}, J=8.5 \mathrm{~Hz}$, $2 \mathrm{H}, \mathrm{ArH}), 6.47(\mathrm{~s}, 1 \mathrm{H}, \mathrm{CH}), 5.01(\mathrm{~d}, J=17.3 \mathrm{~Hz}, 1 \mathrm{H}$, $\left.\mathrm{CH}_{2}\right), 4.83\left(\mathrm{~d}, J=17.5 \mathrm{~Hz}, 1 \mathrm{H}, \mathrm{CH}_{2}\right), 3.84 \sim 4.21(\mathrm{~m}, 2 \mathrm{H}$, $\left.\mathrm{CH}_{2}\right), 3.93 \sim 3.97\left(\mathrm{~m}, 2 \mathrm{H}, \mathrm{CH}_{2}\right) ;{ }^{13} \mathrm{C}$ NMR $(125 \mathrm{MHz}$, DMSO- $\left.d_{6}\right) \delta: 179.4,156.6,155.6,147.3,144.7,136.6$, $130.3,128.8,127.4,124.0,123.5,123.2,118.6,112.4$, $110.3,85.9,55.1,51.3,45.7$; IR (KBr) v: 1662, 1615, 1552, 1513, 1342, 1318, 1295, 1219, $622 \mathrm{~cm}^{-1}$; HRMS (TOF $\mathrm{ES}^{+}$) calcd for $\mathrm{C}_{21} \mathrm{H}_{17} \mathrm{~N}_{4} \mathrm{O}_{6}[\mathrm{M}+\mathrm{H}]^{+}$421.1143, found 421.1140 .

4-硝基-3-(4-(三氟甲基)芐基)-1,2,3,11 $a$-四氢- $6 H$-苯 并吡喃并 [3,2-e]咪唑并 $[1,2-a]$ 吡啶-6-酮(3ab): 黄色固 体, m.p. $240 \sim 241{ }^{\circ} \mathrm{C} ;{ }^{1} \mathrm{H}$ NMR (500 MHz, DMSO- $d_{6}$ ) $\delta$ : $7.93(\mathrm{~s}, 1 \mathrm{H}, \mathrm{CH}), 7.83 \sim 7.84(\mathrm{~m}, 1 \mathrm{H}, \mathrm{ArH}), 7.75 \sim 7.77$ (m, $2 \mathrm{H}, \operatorname{ArH}), 7.62 \sim 7.63(\mathrm{~m}, 3 \mathrm{H}, \mathrm{ArH}), 7.17 \sim 7.20(\mathrm{~m}$, $1 \mathrm{H}, \mathrm{ArH}), 7.11$ (d, $J=8.0 \mathrm{~Hz}, 1 \mathrm{H}, \mathrm{ArH}), 6.46(\mathrm{~s}, 1 \mathrm{H}, \mathrm{CH})$, $4.97\left(\mathrm{~d}, J=16.5 \mathrm{~Hz}, 1 \mathrm{H}, \mathrm{CH}_{2}\right), 4.80(\mathrm{~d}, J=16.5 \mathrm{~Hz}, 1 \mathrm{H}$, $\left.\mathrm{CH}_{2}\right), 4.16 \sim 4.20\left(\mathrm{~m}, 1 \mathrm{H}, \mathrm{CH}_{2}\right), 3.91 \sim 3.95\left(\mathrm{~m}, 2 \mathrm{H}, \mathrm{CH}_{2}\right)$, $3.83 \sim 3.87\left(\mathrm{~m}, 1 \mathrm{H}, \mathrm{CH}_{2}\right) ;{ }^{13} \mathrm{C}$ NMR $\left(125 \mathrm{MHz}\right.$, DMSO- $\left.d_{6}\right)$ $\delta: 179.3,156.6,155.5,141.4,136.5,130.4,128.5(\mathrm{~d}$, $\left.{ }^{2} J_{\mathrm{C}-\mathrm{F}}=32.5 \mathrm{~Hz}\right), 128.5,127.4,125.8\left(\mathrm{~d},{ }^{3} J_{\mathrm{C}-\mathrm{F}}=3.8 \mathrm{~Hz}\right)$, $123.2 \sim 123.7(\mathrm{~m}), 118.6,112.3,110.3,85.9,55.0,51.2$, 45.6; IR (KBr) v: 1657, 1616, 1557, 1460, 1326, 1295, $1114,618 \mathrm{~cm}^{-1}$; HRMS (TOF ES ${ }^{+}$) calcd for $\mathrm{C}_{22} \mathrm{H}_{17} \mathrm{~F}_{3} \mathrm{~N}_{3}$ $\mathrm{O}_{4}[\mathrm{M}+\mathrm{H}]^{+}$444.1166, found 444.1167.

4-((4-硝基-6-氧代-1,2-二氢-6 $\mathrm{H}$-色烯并 [3,2-e]咪唑 并 $[1,2-a]$ 吡啶-3(11aH)-基)甲基)苯甲腈(3ac): 黄色固体, m.p. $243 \sim 244{ }^{\circ} \mathrm{C}$; ${ }^{1} \mathrm{H}$ NMR (500 MHz, DMSO- $d_{6}$ ) $\delta$ : 7.91 (s, $1 \mathrm{H}, \mathrm{CH}), 7.83 \sim 7.87(\mathrm{~m}, 3 \mathrm{H}, \mathrm{ArH}), 7.59 \sim 7.64$ (m, 3H, ArH), $7.19(\mathrm{~d}, J=7.5 \mathrm{~Hz}, 1 \mathrm{H}, \mathrm{ArH}), 7.11(\mathrm{~d}, J=$ $8.0 \mathrm{~Hz}, 1 \mathrm{H}, \mathrm{ArH}), 6.46$ (s, 1H, CH), 4.96 (d, $J=16.5 \mathrm{~Hz}$, 
$\left.1 \mathrm{H}, \mathrm{CH}_{2}\right), 4.79\left(\mathrm{~d}, J=17.0 \mathrm{~Hz}, 1 \mathrm{H}, \mathrm{CH}_{2}\right), 4.18 \sim 4.19(\mathrm{~m}$, $\left.1 \mathrm{H}, \mathrm{CH}_{2}\right), 3.91 \sim 3.95\left(\mathrm{~m}, 2 \mathrm{H}, \mathrm{CH}_{2}\right), 3.85\left(\mathrm{~m}, 1 \mathrm{H}, \mathrm{CH}_{2}\right)$; ${ }^{13} \mathrm{C}$ NMR $\left(125 \mathrm{MHz}, \mathrm{DMSO}-d_{6}\right) \delta: 179.3,156.6,155.5$, $142.5,136.6,132.8,130.4,128.6,127.4,123.5,123.2$, $119.2,118.6,112.3,110.6,110.3,85.9,55.2,51.3,45.6$; IR (KBr) v: 2222, 1661, 1615, 1553, 1460, 1307, 1293, 1220, $694 \mathrm{~cm}^{-1}$; HRMS (TOF ES ${ }^{+}$) calcd for $\mathrm{C}_{22} \mathrm{H}_{17} \mathrm{~N}_{4} \mathrm{O}_{4}[\mathrm{M}+$ $\mathrm{H}^{+}$401.1244, found 401.1243.

3-(4-氟苄基)-4-硝基-1,2,3,11a-四氢- $6 H$-苯并吡喃并 [3,2-e]咪唑并 $[1,2-a]$ 吡啶-6-酮 (3ad): 黄色固体, m.p. 234 $235{ }^{\circ} \mathrm{C} ;{ }^{1} \mathrm{H}$ NMR (500 MHz, DMSO- $\left.d_{6}\right) \delta: 7.92$ (s, $1 \mathrm{H}, \mathrm{CH}), 7.82 \sim 7.84(\mathrm{~m}, 1 \mathrm{H}, \mathrm{ArH}), 7.60 \sim 7.64(\mathrm{~m}, 1 \mathrm{H}$, ArH $), 7.43 \sim 7.46(\mathrm{~m}, 2 \mathrm{H}, \mathrm{ArH}), 7.16 \sim 7.24(\mathrm{~m}, 3 \mathrm{H}$, $\operatorname{ArH}), 7.10$ (d, $J=8.0 \mathrm{~Hz}, 1 \mathrm{H}, \mathrm{ArH}), 6.43$ (s, 1H, CH), 4.86 $\left(\mathrm{d}, J=15.5 \mathrm{~Hz}, 1 \mathrm{H}, \mathrm{CH}_{2}\right), 4.72\left(\mathrm{~d}, J=15.5 \mathrm{~Hz}, 1 \mathrm{H}, \mathrm{CH}_{2}\right)$, $4.14 \sim 4.16\left(\mathrm{~m}, 1 \mathrm{H}, \mathrm{CH}_{2}\right), 3.87 \sim 3.91\left(\mathrm{~m}, 2 \mathrm{H}, \mathrm{CH}_{2}\right)$, $3.81 \sim 3.83\left(\mathrm{~m}, 1 \mathrm{H}, \mathrm{CH}_{2}\right) ;{ }^{13} \mathrm{C} \mathrm{NMR}\left(125 \mathrm{MHz}, \mathrm{DMSO}-d_{6}\right)$ $\delta: 179.3,162.1\left(\mathrm{~d},{ }^{1} J_{\mathrm{C}-\mathrm{F}}=241.2 \mathrm{~Hz}\right), 156.6,155.1,136.5$, $132.2,130.2\left(\mathrm{~d},{ }^{3} J_{\mathrm{C}-\mathrm{F}}=8.8 \mathrm{~Hz}\right), 127.4,123.5,123.2,118.6$, $115.8\left(\mathrm{~d},{ }^{2} J_{\mathrm{C}-\mathrm{F}}=21.3 \mathrm{~Hz}\right), 112.2,110.4,85.9,54.3,50.8$, 45.5; IR (KBr) v: 3902, 1662, 1609, 1578, 1513, 1290, 1260, 1211, 1188,624 $\mathrm{cm}^{-1}$; HRMS (TOF ES ${ }^{+}$) calcd for $\mathrm{C}_{21} \mathrm{H}_{17} \mathrm{FN}_{3} \mathrm{O}_{4}[\mathrm{M}+\mathrm{H}]^{+}$394.1198, found 394.1201.

3-(4-氯苄基)-4-硝基-1,2,3,11a-四氢- $6 H$-色烯并 [3,2-e]咪唑并 $[1,2-a]$ 吡啶-6-酮 (3ae): 黄色固体, m.p. $249 \sim 250{ }^{\circ} \mathrm{C}$; ${ }^{1} \mathrm{H}$ NMR $\left(500 \mathrm{MHz}, \mathrm{DMSO}-d_{6}\right) \delta: 7.91$ (s, $1 \mathrm{H}, \mathrm{CH}), 7.82 \sim 7.84(\mathrm{~m}, 1 \mathrm{H}, \mathrm{ArH}), 7.61 \sim 7.64(\mathrm{~m}, 1 \mathrm{H}$, ArH), $7.41 \sim 7.46(\mathrm{~m}, 4 \mathrm{H}, \mathrm{ArH}), 7.17 \sim 7.20(\mathrm{~m}, 1 \mathrm{H}, \mathrm{ArH})$, $7.10(\mathrm{~d}, J=8.0 \mathrm{~Hz}, 1 \mathrm{H}, \operatorname{ArH}), 6.44$ (s, 1H, CH), 4.86 (d, $\left.J=16.0 \mathrm{~Hz}, 1 \mathrm{H}, \mathrm{CH}_{2}\right), 4.71\left(\mathrm{~d}, J=16.0 \mathrm{~Hz}, 1 \mathrm{H}, \mathrm{CH}_{2}\right)$, $4.15 \sim 4.17\left(\mathrm{~m}, 1 \mathrm{H}, \mathrm{CH}_{2}\right), 3.88 \sim 3.92\left(\mathrm{~m}, 2 \mathrm{H}, \mathrm{CH}_{2}\right)$, $3.80 \sim 3.84\left(\mathrm{~m}, 1 \mathrm{H}, \mathrm{CH}_{2}\right) ;{ }^{13} \mathrm{C}$ NMR $\left(125 \mathrm{MHz}, \mathrm{DMSO}-d_{6}\right)$ $\delta: 179.3,156.6,155.2,136.6,135.3,132.6,130.4,129.9$, $128.9,127.4,123.5,123.2,118.6,112.3,110.3,85.9,54.4$, 51.0, 45.5; IR (KBr) v: 2975, 1663, 1612, 1462, 1289, 1211, 1187, $622 \mathrm{~cm}^{-1}$; HRMS (TOF ES ${ }^{+}$) calcd for $\mathrm{C}_{21} \mathrm{H}_{17} \mathrm{ClN}_{3} \mathrm{O}_{4}[\mathrm{M}+\mathrm{H}]^{+}$410.0902, found 410.0900.

3- 苄基-4-硝基-1,2,3,11a-四氢- $6 H$ - 苯并吡喃并 [3,2-e]味唑并 [1,2- $a$ 吡啶-6-酮 (3af): 黄色固体, m.p. $242 \sim 243{ }^{\circ} \mathrm{C} ;{ }^{1} \mathrm{H}$ NMR (500 MHz, DMSO- $\left.d_{6}\right) \delta$ : 7.91 (s, $1 \mathrm{H}, \mathrm{CH}), 7.82 \sim 7.84(\mathrm{~m}, 1 \mathrm{H}, \mathrm{ArH}), 7.60 \sim 7.64(\mathrm{~m}, 1 \mathrm{H}$, $\operatorname{ArH}), 7.31 \sim 7.41(\mathrm{~m}, 4 \mathrm{H}, \mathrm{ArH}), 7.16 \sim 7.19(\mathrm{~m}, 1 \mathrm{H}, \mathrm{ArH})$, $7.10(\mathrm{~d}, J=8.5 \mathrm{~Hz}, 1 \mathrm{H}, \mathrm{ArH}), 6.44$ (s, 1H, CH), 4.88 (d, $\left.J=15.5 \mathrm{~Hz}, 1 \mathrm{H}, \mathrm{CH}_{2}\right), 4.75\left(\mathrm{~d}, J=16.0 \mathrm{~Hz}, 1 \mathrm{H}, \mathrm{CH}_{2}\right)$, $4.15 \sim 4.16\left(\mathrm{~m}, 1 \mathrm{H}, \mathrm{CH}_{2}\right), 3.88 \sim 3.92\left(\mathrm{~m}, 2 \mathrm{H}, \mathrm{CH}_{2}\right)$,
$3.79 \sim 3.83\left(\mathrm{~m}, 1 \mathrm{H}, \mathrm{CH}_{2}\right) ;{ }^{13} \mathrm{C}$ NMR (125 MHz, DMSO- $\left.d_{6}\right)$ $\delta: 179.3,156.6,155.1,136.5,136.1,130.5,129.0,128.1$, $128.1,127.4,123.6,123.2,118.6,112.2,110.4,85.9,54.9$, 51.0, 45.5; IR (KBr) v: 3077, 1662, 1612, 1462, 1287, 1211, 1189, 1146, 1035, $625 \mathrm{~cm}^{-1}$; HRMS (TOF ES ${ }^{+}$) calcd for $\mathrm{C}_{21} \mathrm{H}_{18} \mathrm{~N}_{3} \mathrm{O}_{4}[\mathrm{M}+\mathrm{H}]^{+}$376.1292, found 376.1291 .

3-(4-甲基苄基)-4-硝基-1,2,3,11 $a$-四氢- $6 H$-苯并吡喃 并 [3,2-e]味唑并 $[1,2-a]$ 吡啶-6-酮(3ag): 黄色固体, m.p. $219.5 \sim 220{ }^{\circ} \mathrm{C} ;{ }^{1} \mathrm{H}$ NMR (500 MHz, DMSO- $\left.d_{6}\right) \delta: 7.91$ $(\mathrm{s}, 1 \mathrm{H}, \mathrm{CH}), 7.82 \sim 7.84(\mathrm{~m}, 1 \mathrm{H}, \mathrm{ArH}), 7.60 \sim 7.63(\mathrm{~m}, 1 \mathrm{H}$, ArH), $7.26(\mathrm{~d}, J=8.0 \mathrm{~Hz}, 2 \mathrm{H}, \mathrm{ArH}), 7.16 \sim 7.20(\mathrm{~m}, 3 \mathrm{H}$, ArH), 7.09 (d, $J=8.0 \mathrm{~Hz}, 1 \mathrm{H}, \mathrm{ArH}), 6.42$ (s, 1H, CH), 4.82 (d, $\left.J=15.5 \mathrm{~Hz}, 1 \mathrm{H}, \mathrm{CH}_{2}\right), 4.71\left(\mathrm{~d}, J=15.5 \mathrm{~Hz}, 1 \mathrm{H}, \mathrm{CH}_{2}\right.$ ), $4.13 \sim 4.14\left(\mathrm{~m}, 1 \mathrm{H}, \mathrm{CH}_{2}\right), 3.86 \sim 3.90\left(\mathrm{~m}, 2 \mathrm{H}, \mathrm{CH}_{2}\right), 3.79$ $\left(\mathrm{m}, 1 \mathrm{H}, \mathrm{CH}_{2}\right), 2.30\left(\mathrm{~s}, 3 \mathrm{H}, \mathrm{CH}_{3}\right) ;{ }^{13} \mathrm{C} \mathrm{NMR}(125 \mathrm{MHz}$, DMSO- $\left.d_{6}\right) \delta: 179.3,156.6,154.9,136.4,136.5,132.9$, $130.6,129.6,128.2,127.3,123.6,123.2,118.6,112.1$, $110.5,85.9,54.5,50.8,45.4,21.2$; IR (KBr) v: 3075, 1662, $1611,1557,1462,1288,1211,11190,1145,624 \mathrm{~cm}^{-1}$; HRMS (TOF ES ${ }^{+}$) calcd for $\mathrm{C}_{22} \mathrm{H}_{20} \mathrm{~N}_{3} \mathrm{O}_{4}[\mathrm{M}+\mathrm{H}]^{+}$ 390.1448 , found 390.1448 .

3-(4-甲氧基苄基)-4-硝基-1,2,3,11 $a$-四氢- $6 H$-苯并吡 喃并 [3,2-e $]$ 咪唑并 $[1,2-a]$ 吡啶-6-酮 (3ah): 黄色固体, m.p. 229 $230{ }^{\circ} \mathrm{C} ;{ }^{1} \mathrm{H}$ NMR (600 MHz, DMSO-d $) \delta$ : $7.91(\mathrm{~s}, 1 \mathrm{H}, \mathrm{CH}), 7.82 \sim 7.83(\mathrm{~m}, 1 \mathrm{H}, \mathrm{ArH}), 7.60 \sim 7.63$ (m, 1H, ArH), 7.31 (d, $J=8.3 \mathrm{~Hz}, 2 \mathrm{H}, \mathrm{ArH}), 7.16 \sim 7.19$ $(\mathrm{m}, 1 \mathrm{H}, \mathrm{ArH}), 7.08 \sim 7.10(\mathrm{~m}, 1 \mathrm{H}, \operatorname{ArH}), 6.95$ (d, $J=8.5$ $\mathrm{Hz}, 2 \mathrm{H}, \mathrm{ArH}), 6.42$ (s, 1H, CH), 4.79 (d, J=15.2 Hz, 1H, $\left.\mathrm{CH}_{2}\right), 4.69\left(\mathrm{~d}, J=15.2 \mathrm{~Hz}, 1 \mathrm{H}, \mathrm{CH}_{2}\right), 4.11 \sim 4.15(\mathrm{~m}, 1 \mathrm{H}$, $\left.\mathrm{CH}_{2}\right), 3.85 \sim 3.89\left(\mathrm{~m}, 2 \mathrm{H}, \mathrm{CH}_{2}\right), 3.78 \sim 3.79\left(\mathrm{~m}, 1 \mathrm{H}, \mathrm{CH}_{2}\right)$, $3.75\left(\mathrm{~s}, 3 \mathrm{H}, \mathrm{CH}_{3}\right) ;{ }^{13} \mathrm{C} \mathrm{NMR}\left(150 \mathrm{MHz}, \mathrm{DMSO}-d_{6}\right) \delta$ : $179.3,159.4,156.6,154.8,136.5,130.6,129.7,127.7$, $127.3,123.5,123.2,118.6,114.5,112.2,110.5,85.9,55.6$, 54.1, 50.6, 45.4; IR (KBr) v: 3075, 2833, 1660, 1611, $1580,1515,1305,1259,1210,1188,623 \mathrm{~cm}^{-1}$; HRMS (TOF $\mathrm{ES}^{+}$) calcd for $\mathrm{C}_{22} \mathrm{H}_{20} \mathrm{~N}_{3} \mathrm{O}_{5}[\mathrm{M}+\mathrm{H}]^{+}$406.1397, found 406.1398 .

3-(3-氟苠基)-4-硝基-1,2,3,11a-四氢- $6 H$-苯并吡喃并 [3,2-e]味唑并 [1,2- $a$ ]吡啶 -6-酮 (3ai): 黄色固体, m.p. $218 \sim 219{ }^{\circ} \mathrm{C} ;{ }^{1} \mathrm{H}$ NMR (500 MHz, DMSO- $\left.d_{6}\right) \delta: 7.92(\mathrm{~s}$, $1 \mathrm{H}, \mathrm{CH}), 7.83 \sim 7.85(\mathrm{~m}, 1 \mathrm{H}, \mathrm{ArH}), 7.61 \sim 7.64(\mathrm{~m}, 1 \mathrm{H}$, ArH), $7.42 \sim 7.46(\mathrm{~m}, 1 \mathrm{H}, \mathrm{ArH}), 7.27$ (d, $J=10.0 \mathrm{~Hz}, 1 \mathrm{H}$, ArH), $7.14 \sim 7.23(\mathrm{~m}, 2 \mathrm{H}, \operatorname{ArH}), 7.11(\mathrm{~d}, J=8.0 \mathrm{~Hz}, 1 \mathrm{H}$, ArH), 6.44 (s, 1H, CH), 4.90 (d, $J=16.0 \mathrm{~Hz}, 1 \mathrm{H}, \mathrm{CH}_{2}$ ), 
$4.72\left(\mathrm{~d}, J=16.0 \mathrm{~Hz}, 1 \mathrm{H}, \mathrm{CH}_{2}\right), 4.15 \sim 4.18\left(\mathrm{~m}, 1 \mathrm{H}, \mathrm{CH}_{2}\right)$, $3.90 \sim 3.94\left(\mathrm{~m}, 2 \mathrm{H}, \mathrm{CH}_{2}\right), 3.81 \sim 3.85\left(\mathrm{~m}, 1 \mathrm{H}, \mathrm{CH}_{2}\right) ;{ }^{13} \mathrm{C}$ NMR $\left(125 \mathrm{MHz}\right.$, DMSO- $\left.d_{6}\right) \delta: 179.3,162.8\left(\mathrm{~d},{ }^{1} J_{\mathrm{C}-\mathrm{F}}=\right.$ $241.3 \mathrm{~Hz}), 156.6,155.3,139.2\left(\mathrm{~d},{ }^{3} J_{\mathrm{C}-\mathrm{F}}=6.3 \mathrm{~Hz}\right), 136.5$, $130.9\left(\mathrm{~d},{ }^{3} J_{\mathrm{C}-\mathrm{F}}=8.8 \mathrm{~Hz}\right), 130.5,127.4,123.9,123.5,123.2$, $118.6,114.9,114.8\left(\mathrm{~d},{ }^{2} J_{\mathrm{C}-\mathrm{F}}=21.3 \mathrm{~Hz}\right), 112.3,110.4,85.9$, 54.7, 51.1, 45.5; IR (KBr) v: 3071, 1608, 1578, 1513, $1289,1259,1210,1186,1145,623 \mathrm{~cm}^{-1}$; HRMS (TOF $\mathrm{ES}^{+}$) calcd for $\mathrm{C}_{21} \mathrm{H}_{17} \mathrm{FN}_{3} \mathrm{O}_{4}[\mathrm{M}+\mathrm{H}]^{+}$394.1198, found 394.1201.

3-(3,5-二氟苄基)-4-硝基-1,2,3,11 $a$-四氢- $6 H$-苯并吡 喃并[3,2-e]咪唑并 $[1,2-a]$ 吡啶-6-酮(3aj): 黄色固体, m.p. $243.5 \sim 244{ }^{\circ} \mathrm{C} ;{ }^{1} \mathrm{H}$ NMR (500 MHz, DMSO- $\left.d_{6}\right) \delta: 7.92$ (s, $1 \mathrm{H}, \mathrm{CH}), 7.83 \sim 7.85(\mathrm{~m}, 1 \mathrm{H}, \mathrm{ArH}), 7.61 \sim 7.63(\mathrm{~m}, 1 \mathrm{H}$, ArH), $7.16 \sim 7.20(\mathrm{~m}, 3 \mathrm{H}, \mathrm{ArH}), 7.11(\mathrm{~d}, J=8.5 \mathrm{~Hz}, 1 \mathrm{H}$, $\mathrm{ArH}), 6.45$ (s, 1H, CH), 4.89 (d, J=16.5 Hz, 1H, $\mathrm{CH}_{2}$ ), $4.68\left(\mathrm{~d}, J=17.0 \mathrm{~Hz}, 1 \mathrm{H}, \mathrm{CH}_{2}\right), 4.17 \sim 4.19\left(\mathrm{~m}, 1 \mathrm{H}, \mathrm{CH}_{2}\right)$, $3.91 \sim 3.95\left(\mathrm{~m}, 2 \mathrm{H}, \mathrm{CH}_{2}\right), 3.84\left(\mathrm{~m}, 1 \mathrm{H}, \mathrm{CH}_{2}\right) ;{ }^{13} \mathrm{C} \mathrm{NMR}$ $\left(125 \mathrm{MHz}, \mathrm{DMSO}-d_{6}\right) \delta: 179.3,162.9\left(\mathrm{dd},{ }^{1} J_{\mathrm{C}-\mathrm{F}}=246.3\right.$ $\left.\mathrm{Hz},{ }^{3} J_{\mathrm{C}-\mathrm{F}}=13.8 \mathrm{~Hz}\right), 156.6,155.5,141.4,136.6,130.4$, $127.4,123.6,123.2,118.6,112.4,111.0\left(\mathrm{~d},{ }^{2} J_{\mathrm{C}-\mathrm{F}}=25.0\right.$ $\mathrm{Hz}), 110.3,103.3\left(\mathrm{t},{ }^{2} J_{\mathrm{C}-\mathrm{F}}=26.3 \mathrm{~Hz}\right), 85.9,54.8,51.2$, 45.6; IR (KBr) v: 3076, 1665, 1619, 1551, 1452, 1272, 1222, $616 \mathrm{~cm}^{-1}$; HRMS (TOF $\mathrm{ES}^{+}$) calcd for $\mathrm{C}_{21} \mathrm{H}_{16} \mathrm{~F}_{2}$ $\mathrm{N}_{3} \mathrm{O}_{4}[\mathrm{M}+\mathrm{H}]^{+}$412.1103, found 412.1103 .

3-((6-氯吡啶-3-基)甲基)-4-硝基-1,2,3,11a-四氢- $6 \mathrm{H}$ 苯并吡喃并 $[3,2-e]$ 咪唑并 $[1,2-a]$ 吡啶-6-酮(3ak): 黄色固 体, m.p. 230 231 ${ }^{\circ} \mathrm{C} ;{ }^{1} \mathrm{H}$ NMR (500 MHz, DMSO- $\left.d_{6}\right) \delta$ : $8.46(\mathrm{~d}, J=2.0 \mathrm{~Hz}, 1 \mathrm{H}, \mathrm{CH}), 7.90 \sim 7.92(\mathrm{~m}, 2 \mathrm{H}, \mathrm{ArH})$, $7.83 \sim 7.84$ (m, 1H, ArH), $7.61 \sim 7.64$ (m, 1H, ArH), 7.55 (d, $J=8.5 \mathrm{~Hz}, 1 \mathrm{H}, \mathrm{ArH}), 7.17 \sim 7.20(\mathrm{~m}, 1 \mathrm{H}, \mathrm{ArH}), 7.10$ (d, $J=8.0 \mathrm{~Hz}, 1 \mathrm{H}, \mathrm{ArH}), 6.44$ (s, 1H, CH), 4.90 (d, $J=$ $\left.16.5 \mathrm{~Hz}, 1 \mathrm{H}, \mathrm{CH}_{2}\right), 4.72\left(\mathrm{~d}, J=16.5 \mathrm{~Hz}, 1 \mathrm{H}, \mathrm{CH}_{2}\right), 4.15 \sim$ $4.20\left(\mathrm{~m}, 1 \mathrm{H}, \mathrm{CH}_{2}\right), 3.92 \sim 3.96\left(\mathrm{~m}, 2 \mathrm{H}, \mathrm{CH}_{2}\right), 3.79 \sim 3.85$ $\left(\mathrm{m}, 1 \mathrm{H}, \mathrm{CH}_{2}\right) ;{ }^{13} \mathrm{C}$ NMR $\left(125 \mathrm{MHz}\right.$, DMSO- $\left.d_{6}\right) \delta: 179.3$, $156.6,155.5,149.9,149.5,139.5,136.5,131.8,130.3$, $127.4,124.5,123.5,123.2,118.6,112.4,110.3,85.9,52.6$, 51.1, 45.6; IR (KBr) v: 1659, 1614, 1549, 1460, 1328, 1293, 1278, 1219, $622 \mathrm{~cm}^{-1}$; HRMS (TOF $\mathrm{ES}^{+}$) calcd for $\mathrm{C}_{20} \mathrm{H}_{16} \mathrm{ClN}_{4} \mathrm{O}_{4}[\mathrm{M}+\mathrm{H}]^{+}$411.0855, found 411.0854.

3 -((2-氯噻唑-5-基)甲基)-4-硝基-1,2,3,11a-四氢- $6 H$ 苯并吡喃并[3,2-e]咪唑并[1,2- $a$ 吡啶-6-酮(3al): 黄色固 体, m.p. $258 \sim 259{ }^{\circ} \mathrm{C} ;{ }^{1} \mathrm{H}$ NMR (500 MHz, DMSO- $\left.d_{6}\right) \delta$ : $7.92(\mathrm{~s}, 1 \mathrm{H}, \mathrm{CH}), 7.82 \sim 7.84(\mathrm{~m}, 1 \mathrm{H}, \mathrm{ArH}), 7.78(\mathrm{~s}, 1 \mathrm{H}$, $\mathrm{ArH}), 7.61 \sim 7.64(\mathrm{~m}, 1 \mathrm{H}, \mathrm{ArH}), 7.17 \sim 7.20(\mathrm{~s}, 1 \mathrm{H}, \mathrm{ArH})$, $7.10(\mathrm{~d}, J=8.0 \mathrm{~Hz}, 1 \mathrm{H}, \mathrm{ArH}), 6.43$ (s, 1H, CH), 4.99 (d, $\left.J=16.0 \mathrm{~Hz}, 1 \mathrm{H}, \mathrm{CH}_{2}\right), 4.83\left(\mathrm{~d}, J=16.0 \mathrm{~Hz}, 1 \mathrm{H}, \mathrm{CH}_{2}\right)$, $4.15 \sim 4.16\left(\mathrm{~m}, 1 \mathrm{H}, \mathrm{CH}_{2}\right), 3.88 \sim 3.96\left(\mathrm{~m}, 2 \mathrm{H}, \mathrm{CH}_{2}\right)$, $3.77 \sim 3.79\left(\mathrm{~m}, 1 \mathrm{H}, \mathrm{CH}_{2}\right) ;{ }^{13} \mathrm{C}$ NMR $\left(125 \mathrm{MHz}\right.$, DMSO- $\left.d_{6}\right)$ $\delta: 179.3,156.5,154.8,152.3,142.2,136.7,135.7,130.0$, 127.4, 123.4, 123.3, 118.6, 112.9, 110.3, 85.7, 50.4, 48.6, 45.4; IR (KBr) v: 1613, 1563,1294, 1267, 1215, 1052, 619 $\mathrm{cm}^{-1}$; HRMS (TOF ES ${ }^{+}$) calcd for $\mathrm{C}_{18} \mathrm{H}_{14} \mathrm{ClN}_{4} \mathrm{O}_{4} \mathrm{~S}$ [M+ $\mathrm{H}]^{+}$417.0419, found 417.0417 .

8-氟-4-硝基-3-(4-(三氟甲基)芐基)-1,2,3,11 $a$-四氢$6 H$-苯并吡喃并 $[3,2-e]$ 咪唑并 $[1,2-a]$ 吡啶-6-酮(3bb): 黄 色固体, m.p. $233 \sim 234{ }^{\circ} \mathrm{C} ;{ }^{1} \mathrm{H}$ NMR $(500 \mathrm{MHz}$, DMSO- $\left.d_{6}\right) \delta: 7.95(\mathrm{~s}, 1 \mathrm{H}, \mathrm{CH}), 7.56(\mathrm{~d}, J=8.0 \mathrm{~Hz}, 2 \mathrm{H}$, $\operatorname{ArH}), 7.63(\mathrm{~d}, J=8.0 \mathrm{~Hz}, 2 \mathrm{H}, \operatorname{ArH}), 7.49 \sim 7.53(\mathrm{~m}, 2 \mathrm{H}$, $\mathrm{ArH}), 7.16 \sim 7.18(\mathrm{~m}, 1 \mathrm{H}, \mathrm{ArH}), 6.45(\mathrm{~s}, 1 \mathrm{H}, \mathrm{CH}), 4.97(\mathrm{~d}$, $\left.J=16.5 \mathrm{~Hz}, 1 \mathrm{H}, \mathrm{CH}_{2}\right), 4.81\left(\mathrm{~d}, J=16.5 \mathrm{~Hz}, 1 \mathrm{H}, \mathrm{CH}_{2}\right)$, $4.15 \sim 4.20\left(\mathrm{~m}, 1 \mathrm{H}, \mathrm{CH}_{2}\right), 3.92 \sim 3.95\left(\mathrm{~m}, 2 \mathrm{H}, \mathrm{CH}_{2}\right)$, $3.81 \sim 3.87\left(\mathrm{~m}, 1 \mathrm{H}, \mathrm{CH}_{2}\right) ;{ }^{13} \mathrm{C}$ NMR $\left(125 \mathrm{MHz}\right.$, DMSO- $\left.d_{6}\right)$ $\delta: 178.5,157.8\left(\mathrm{~d},{ }^{1} J_{\mathrm{C}-\mathrm{F}}=238.8 \mathrm{~Hz}\right), 155.4,152.8,141.3$, $131.1,128.5\left(\mathrm{~d},{ }^{2} J_{\mathrm{C}-\mathrm{F}}=31.3 \mathrm{~Hz}\right), 128.5,125.7\left(\mathrm{~d},{ }^{3} J_{\mathrm{C}-\mathrm{F}}=\right.$ $7.5 \mathrm{~Hz}), 124.4\left(\mathrm{~d},{ }^{3} J_{\mathrm{C}-\mathrm{F}}=7.5 \mathrm{~Hz}\right), 123.6,123.7\left(\mathrm{~d},{ }^{2} J_{\mathrm{C}-\mathrm{F}}=\right.$ $23.8 \mathrm{~Hz}), 120.8\left(\mathrm{~d},{ }^{3} J_{\mathrm{C}-\mathrm{F}}=7.5 \mathrm{~Hz}\right), 112.3\left(\mathrm{~d},{ }^{2} J_{\mathrm{C}-\mathrm{F}}=23.8\right.$ $\mathrm{Hz}), 111.5,110.5,86.0,55.0,51.2$, 45.6; IR (KBr) v: 2891, $1656,1601,1555,1479,1324,1275,1258,1126,1065$, $631 \mathrm{~cm}^{-1}$; HRMS (TOF $\mathrm{ES}^{+}$) calcd for $\mathrm{C}_{22} \mathrm{H}_{16} \mathrm{~F}_{4} \mathrm{~N}_{3} \mathrm{O}_{4}$ $[\mathrm{M}+\mathrm{H}]^{+}$462.1071, found 462.1069 .

4-((8-氟-4-硝基-6-氧代-1,2-二氢- $6 H$-色烯并 [3,2-e] 咪唑并 $[1,2-a$ 吡啶-3(11aH)-基)甲基)苯甲腈(3bc)：黄色 固体, m.p. $258 \sim 259{ }^{\circ} \mathrm{C} ;{ }^{1} \mathrm{H}$ NMR (500 MHz, DMSO- $d_{6}$ ) $\delta: 7.93(\mathrm{~s}, 1 \mathrm{H}, \mathrm{CH}), 7.87$ (d, $J=8.5 \mathrm{~Hz}, 2 \mathrm{H}, \mathrm{ArH}), 7.60(\mathrm{~d}$, $J=8.0 \mathrm{~Hz}, 2 \mathrm{H}, \mathrm{ArH}), 7.49 \sim 7.53(\mathrm{~m}, 2 \mathrm{H}, \mathrm{ArH}), 7.16 \sim$ 7.19 (m, 1H, ArH), 6.45 (s, 1H, CH), 4.96 (d, $J=17.0 \mathrm{~Hz}$, $\left.1 \mathrm{H}, \mathrm{CH}_{2}\right), 4.79\left(\mathrm{~d}, J=17.0 \mathrm{~Hz}, 1 \mathrm{H}, \mathrm{CH}_{2}\right), 4.15 \sim 4.20(\mathrm{~m}$, $\left.1 \mathrm{H}, \mathrm{CH}_{2}\right), 3.91 \sim 3.95\left(\mathrm{~m}, 2 \mathrm{H}, \mathrm{CH}_{2}\right), 3.83 \sim 3.87(\mathrm{~m}, 1 \mathrm{H}$, $\left.\mathrm{CH}_{2}\right) ;{ }^{13} \mathrm{C}$ NMR (125 MHz, DMSO- $\left.d_{6}\right) \delta: 178.5,157.8(\mathrm{~d}$, $\left.{ }^{1} J_{\mathrm{C}-\mathrm{F}}=238.8 \mathrm{~Hz}\right), 155.5,152.8,142.4,132.7,131.0,128.6$, $124.4\left(\mathrm{~d},{ }^{3} J_{\mathrm{C}-\mathrm{F}}=7.5 \mathrm{~Hz}\right), 123.7\left(\mathrm{~d},{ }^{2} J_{\mathrm{C}-\mathrm{F}}=25.0 \mathrm{~Hz}\right), 120.8$ $\left(\mathrm{d},{ }^{3} J_{\mathrm{C}-\mathrm{F}}=8.8 \mathrm{~Hz}\right), 119.3,112.3\left(\mathrm{~d},{ }^{2} J_{\mathrm{C}-\mathrm{F}}=22.5 \mathrm{~Hz}\right), 111.5$, 110.6, 110.5, 86.0, 55.2, 51.3, 45.7; IR (KBr) v: 2225, $1656,1603,1554,1479,1317,1280,1258,1218,621$ $\mathrm{cm}^{-1}$; HRMS (TOF ES ${ }^{+}$) calcd for $\mathrm{C}_{22} \mathrm{H}_{16} \mathrm{FN}_{4} \mathrm{O}_{4}[\mathrm{M}+\mathrm{H}]^{+}$ 419.1150, found 419.1151.

8 -氟-3-(4-氟苄基)-4-硝基-1,2,3,11 $a$-四氢- $6 H$-色烯 并[3,2-e]咪唑并 $[1,2-a]$ 吡啶-6-酮(3bd): 黄色固体, m.p. 204 205 ${ }^{\circ} \mathrm{C} ;{ }^{1} \mathrm{H}$ NMR (500 MHz, DMSO- $d_{6}$ ) $\delta: 7.94$ (s, 
$1 \mathrm{H}, \mathrm{CH}), 7.43 \sim 7.52(\mathrm{~m}, 4 \mathrm{H}, \mathrm{ArH}), 7.20 \sim 7.24(\mathrm{~m}, 2 \mathrm{H}$, $\operatorname{ArH}), 7.14 \sim 7.17(\mathrm{~m}, 1 \mathrm{H}, \mathrm{ArH}), 6.42(\mathrm{~s}, 1 \mathrm{H}, \mathrm{CH}), 4.85(\mathrm{~d}$, $\left.J=15.5 \mathrm{~Hz}, 1 \mathrm{H}, \mathrm{CH}_{2}\right), 4.72\left(\mathrm{~d}, J=16.0 \mathrm{~Hz}, 1 \mathrm{H}, \mathrm{CH}_{2}\right)$, $4.12 \sim 4.16\left(\mathrm{~m}, 1 \mathrm{H}, \mathrm{CH}_{2}\right), 3.87 \sim 3.91\left(\mathrm{~m}, 2 \mathrm{H}, \mathrm{CH}_{2}\right)$, $3.76 \sim 3.82\left(\mathrm{~m}, 1 \mathrm{H}, \mathrm{CH}_{2}\right) ;{ }^{13} \mathrm{C}$ NMR $\left(125 \mathrm{MHz}, \mathrm{DMSO}-d_{6}\right)$ $\delta: 178.5,162.1\left(\mathrm{~d},{ }^{1} J_{\mathrm{C}-\mathrm{F}}=242.5 \mathrm{~Hz}\right), 157.8\left(\mathrm{~d},{ }^{1} J_{\mathrm{C}-\mathrm{F}}=\right.$ $238.8 \mathrm{~Hz}), 155.0,152.8,132.2\left(\mathrm{~d},{ }^{4} J_{\mathrm{C}-\mathrm{F}}=2.5 \mathrm{~Hz}\right), 131.2$, $130.2\left(\mathrm{~d},{ }^{3} J_{\mathrm{C}-\mathrm{F}}=7.5 \mathrm{~Hz}\right), 124.4\left(\mathrm{~d},{ }^{3} J_{\mathrm{C}-\mathrm{F}}=6.3 \mathrm{~Hz}\right), 123.7$ $\left(\mathrm{d},{ }^{2} J_{\mathrm{C}-\mathrm{F}}=23.8 \mathrm{~Hz}\right), 120.8\left(\mathrm{~d},{ }^{3} J_{\mathrm{C}-\mathrm{F}}=7.5 \mathrm{~Hz}\right), 115.8(\mathrm{~d}$, $\left.{ }^{2} J_{\mathrm{C}-\mathrm{F}}=21.3 \mathrm{~Hz}\right), 112.3\left(\mathrm{~d},{ }^{2} J_{\mathrm{C}-\mathrm{F}}=23.8 \mathrm{~Hz}\right), 111.4,110.6$, 86.1, 54.3, 50.9, 45.5; IR (KBr) v: 3075, 1667, 1618, 1575, 1476, 1306, 1260, 1187, 952, $618 \mathrm{~cm}^{-1}$; HRMS (TOF $\mathrm{ES}^{+}$) calcd for $\mathrm{C}_{21} \mathrm{H}_{16} \mathrm{~F}_{2} \mathrm{~N}_{3} \mathrm{O}_{4}[\mathrm{M}+\mathrm{H}]^{+}$412.1103, found 412.1102 .

3-(4-氯苄基)-8-氟-4-硝基-1,2,3,11 $a$-四氢- $6 H$-色烯 并 $[3,2-e]$ 咪唑并 $[1,2-a]$ 吡啶-6-酮(3be): 黄色固体, m.p. 236 $237{ }^{\circ} \mathrm{C} ;{ }^{1} \mathrm{H}$ NMR (500 MHz, DMSO- $\left.d_{6}\right) \delta: 7.93(\mathrm{~s}$, $1 \mathrm{H}, \mathrm{CH}), 7.48 \sim 7.53(\mathrm{~m}, 2 \mathrm{H}, \mathrm{ArH}), 7.41 \sim 7.46(\mathrm{~m}, 4 \mathrm{H}$, $\mathrm{ArH}), 7.15 \sim 7.17$ (m, 1H, ArH), $6.42(\mathrm{~s}, 1 \mathrm{H}, \mathrm{CH}), 4.86$ (d, $\left.J=16.0 \mathrm{~Hz}, 1 \mathrm{H}, \mathrm{CH}_{2}\right), 4.71\left(\mathrm{~d}, J=16.0 \mathrm{~Hz}, 1 \mathrm{H}, \mathrm{CH}_{2}\right)$, $4.12 \sim 4.16\left(\mathrm{~m}, 1 \mathrm{H}, \mathrm{CH}_{2}\right), 3.88 \sim 3.92\left(\mathrm{~m}, 2 \mathrm{H}, \mathrm{CH}_{2}\right)$, $3.79 \sim 3.83\left(\mathrm{~m}, 1 \mathrm{H}, \mathrm{CH}_{2}\right) ;{ }^{13} \mathrm{C}$ NMR $\left(125 \mathrm{MHz}, \mathrm{DMSO}-d_{6}\right)$ $\delta: 178.5,157.8\left(\mathrm{~d},{ }^{1} J_{\mathrm{C}-\mathrm{F}}=240.0 \mathrm{~Hz}\right), 155.1,152.8,135.2$, 132.7, 131.1, 129.9, 128.9, $124.4\left(\mathrm{~d},{ }^{3} J_{\mathrm{C}-\mathrm{F}}=6.3 \mathrm{~Hz}\right), 123.7$ $\left(\mathrm{d},{ }^{2} J_{\mathrm{C}-\mathrm{F}}=23.8 \mathrm{~Hz}\right), 120.8\left(\mathrm{~d},{ }^{3} J_{\mathrm{C}-\mathrm{F}}=7.5 \mathrm{~Hz}\right), 112.3(\mathrm{~d}$, $\left.{ }^{2} J_{\mathrm{C}-\mathrm{F}}=23.8 \mathrm{~Hz}\right), 111.4,110.6,86.0,54.4,51.0,45.6$; IR (KBr) $v: 3081,1655,1603,1557,1479,1280,1260,1216$, $619 \mathrm{~cm}^{-1}$; HRMS (TOF $\mathrm{ES}^{+}$) calcd for $\mathrm{C}_{21} \mathrm{H}_{16} \mathrm{ClFN}_{3} \mathrm{O}_{4}$ $[\mathrm{M}+\mathrm{H}]^{+}$428.0808, found 428.0809 .

3 - 苄基-8-氟-4-硝基-1,2,3,11a-四氢- $6 H$-色烯并 [3,2-e]味唑并 [1,2- $a$ 吡啶-6-酮 (3bf): 黄色固体, m.p. $231 \sim 232{ }^{\circ} \mathrm{C} ;{ }^{1} \mathrm{H}$ NMR (500 MHz, DMSO- $\left.d_{6}\right) \delta: 7.94$ (s, $1 \mathrm{H}, \mathrm{CH}), 7.48 \sim 7.52(\mathrm{~m}, 2 \mathrm{H}, \mathrm{ArH}), 7.37 \sim 7.40(\mathrm{~m}, 3 \mathrm{H}$, ArH), $7.33 \sim 7.34(\mathrm{~m}, 1 \mathrm{H}, \mathrm{ArH}), 7.14 \sim 7.17$ (m, 1H, ArH), $6.42(\mathrm{~s}, 1 \mathrm{H}, \mathrm{CH}), 4.88\left(\mathrm{~d}, J=16.0 \mathrm{~Hz}, 1 \mathrm{H}, \mathrm{CH}_{2}\right), 4.75$ (d, $\left.J=15.5 \mathrm{~Hz}, 1 \mathrm{H}, \mathrm{CH}_{2}\right), 4.13 \sim 4.15\left(\mathrm{~m}, 1 \mathrm{H}, \mathrm{CH}_{2}\right), 3.89 \sim$ $3.92\left(\mathrm{~m}, 2 \mathrm{H}, \mathrm{CH}_{2}\right), 3.79 \sim 3.83\left(\mathrm{~m}, 1 \mathrm{H}, \mathrm{CH}_{2}\right)$; ${ }^{13} \mathrm{C} \mathrm{NMR}$ $\left(125 \mathrm{MHz}, \mathrm{DMSO}-d_{6}\right) \delta: 178.5,157.8\left(\mathrm{~d},{ }^{1} J_{\mathrm{C}-\mathrm{F}}=238.8\right.$ $\mathrm{Hz}), 155.0,152.8,136.0,131.2,129.0,128.1\left(\mathrm{~d},{ }^{3} J_{\mathrm{C}-\mathrm{F}}=8.8\right.$ $\mathrm{Hz}), 124.4\left(\mathrm{~d},{ }^{3} J_{\mathrm{C}-\mathrm{F}}=7.5 \mathrm{~Hz}\right), 123.7\left(\mathrm{~d},{ }^{2} J_{\mathrm{C}-\mathrm{F}}=25.0 \mathrm{~Hz}\right)$, $120.8\left(\mathrm{~d},{ }^{3} J_{\mathrm{C}-\mathrm{F}}=7.5 \mathrm{~Hz}\right), 112.3\left(\mathrm{~d},{ }^{2} J_{\mathrm{C}-\mathrm{F}}=22.5 \mathrm{~Hz}\right), 111.3$, 110.7, 86.1, 54.9, 51.0, 45.5; IR (KBr) v: 3068, 1665, $1609,1548,1481,1287,1254,1200,1024,620 \mathrm{~cm}^{-1}$; HRMS (TOF $\mathrm{ES}^{+}$) calcd for $\mathrm{C}_{21} \mathrm{H}_{17} \mathrm{FN}_{3} \mathrm{O}_{4}[\mathrm{M}+\mathrm{H}]^{+}$ 394.1198, found 394.1199.
8-氟-3-(4-甲基苠基)-4-硝基-1,2,3,11 $a$-四氢- $6 H$-色 烯并 [3,2-e]咪唑并 $[1,2-a]$ 吡啶-6-酮 $(\mathbf{3 b g})$ : 黄色固体, m.p. $212 \sim 213{ }^{\circ} \mathrm{C} ;{ }^{1} \mathrm{H}$ NMR (500 MHz, DMSO- $\left.d_{6}\right) \delta$ : $7.93(\mathrm{~s}, 1 \mathrm{H}, \mathrm{CH}), 7.48 \sim 7.52(\mathrm{~m}, 2 \mathrm{H}, \mathrm{ArH}), 7.26(\mathrm{~d}, J=$ $7.5 \mathrm{~Hz}, 2 \mathrm{H}, \mathrm{ArH}), 7.20$ (d, J=8.0 Hz, 2H, ArH), 7.14 7.17 (m, 1H, ArH), $6.41(\mathrm{~s}, 1 \mathrm{H}, \mathrm{CH}), 4.82$ (d, J=15.5 Hz, $\left.1 \mathrm{H}, \mathrm{CH}_{2}\right), 4.71\left(\mathrm{~d}, J=15.5 \mathrm{~Hz}, 1 \mathrm{H}, \mathrm{CH}_{2}\right), 4.12 \sim 4.14(\mathrm{~m}$, $\left.1 \mathrm{H}, \mathrm{CH}_{2}\right), 3.87 \sim 3.91\left(\mathrm{~m}, 2 \mathrm{H}, \mathrm{CH}_{2}\right), 3.77 \sim 3.79(\mathrm{~m}, 1 \mathrm{H}$, $\left.\mathrm{CH}_{2}\right), 2.31\left(\mathrm{~s}, 3 \mathrm{H}, \mathrm{CH}_{3}\right) ;{ }^{13} \mathrm{C} \mathrm{NMR}\left(125 \mathrm{MHz}, \mathrm{DMSO}-d_{6}\right)$ $\delta: 178.5,157.8\left(\mathrm{~d},{ }^{1} J_{\mathrm{C}-\mathrm{F}}=238.8 \mathrm{~Hz}\right), 154.8,152.8,137.4$, $132.9,131.2,129.6,128.2,124.4\left(\mathrm{~d},{ }^{3} J_{\mathrm{C}-\mathrm{F}}=6.3 \mathrm{~Hz}\right), 123.7$ $\left(\mathrm{d},{ }^{2} J_{\mathrm{C}-\mathrm{F}}=23.8 \mathrm{~Hz}\right), 120.8\left(\mathrm{~d},{ }^{3} J_{\mathrm{C}-\mathrm{F}}=7.5 \mathrm{~Hz}\right), 112.3(\mathrm{~d}$, $\left.{ }^{2} J_{\mathrm{C}-\mathrm{F}}=23.8 \mathrm{~Hz}\right), 111.3,110.7,86.1,54.5,50.8,45.5,21.2$; IR (KBr) v: 2924, 1666, 1609, 1575, 1311, 1259, 1187, 619 $\mathrm{cm}^{-1}$; HRMS (TOF ES ${ }^{+}$) calcd for $\mathrm{C}_{22} \mathrm{H}_{19} \mathrm{FN}_{3} \mathrm{O}_{4}[\mathrm{M}+\mathrm{H}]^{+}$ 408.1354, found 408.1354.

8-氟-3-(4-甲氧基芳基)-4-硝基-1,2,3,11a-四氢- $6 H$ 色烯并 [3,2-e]咪唑并 $[1,2-a]$ 吡啶-6-酮(3bh): 黄色固体, m.p. $219 \sim 220{ }^{\circ} \mathrm{C} ;{ }^{1} \mathrm{H}$ NMR $\left(500 \mathrm{MHz}, \mathrm{DMSO}-d_{6}\right) \delta$ : $7.93(\mathrm{~s}, 1 \mathrm{H}, \mathrm{CH}), 7.47 \sim 7.52(\mathrm{~m}, 2 \mathrm{H}, \mathrm{ArH}), 7.30(\mathrm{~d}, J=$ $8.5 \mathrm{~Hz}, 2 \mathrm{H}, \mathrm{ArH}), 7.14 \sim 7.17$ (m, 1H, ArH), 6.95 (d, $J=$ $8.5 \mathrm{~Hz}, 2 \mathrm{H}, \mathrm{ArH}), 6.41$ (s, 1H, CH), 4.79 (d, $J=15.5 \mathrm{~Hz}$, $\left.1 \mathrm{H}, \mathrm{CH}_{2}\right), 4.69\left(\mathrm{~d}, J=15.5 \mathrm{~Hz}, 1 \mathrm{H}, \mathrm{CH}_{2}\right), 4.09 \sim 4.14(\mathrm{~m}$, $\left.1 \mathrm{H}, \mathrm{CH}_{2}\right), 3.85 \sim 3.89\left(\mathrm{~m}, 2 \mathrm{H}, \mathrm{CH}_{2}\right), 3.73 \sim 3.79(\mathrm{~m}, 1 \mathrm{H}$, $\left.\mathrm{CH}_{2}\right), 3.76\left(\mathrm{~s}, 3 \mathrm{H}, \mathrm{CH}_{3}\right) ;{ }^{13} \mathrm{C}$ NMR $\left(125 \mathrm{MHz}, \mathrm{DMSO}-d_{6}\right)$ $\delta: 178.5,159.4,157.8\left(\mathrm{~d},{ }^{1} J_{\mathrm{C}-\mathrm{F}}=238.8 \mathrm{~Hz}\right), 154.7,152.8$, 131.2, 129.7, 127.6, $124.4\left(\mathrm{~d},{ }^{3} J_{\mathrm{C}-\mathrm{F}}=7.5 \mathrm{~Hz}\right), 123.7(\mathrm{~d}$, $\left.{ }^{2} J_{\mathrm{C}-\mathrm{F}}=25.0 \mathrm{~Hz}\right), 120.8\left(\mathrm{~d},{ }^{3} J_{\mathrm{C}-\mathrm{F}}=7.5 \mathrm{~Hz}\right), 114.5,112.3(\mathrm{~d}$, $\left.{ }^{2} J_{\mathrm{C}-\mathrm{F}}=23.8 \mathrm{~Hz}\right), 111.3,110.7,86.1,55.6,54.1,50.7,45.4$; IR (KBr) v: 3073, 1662, 1568, 1510, 1443, 1267, 1212, $617 \mathrm{~cm}^{-1}$; HRMS (TOF ES ${ }^{+}$) calcd for $\mathrm{C}_{22} \mathrm{H}_{19} \mathrm{FN}_{3} \mathrm{O}_{5}[\mathrm{M}+$ $\mathrm{H}]^{+}$424.1303, found 424.1305.

8-氟-3-(3-氟苄基)-4-硝基-1,2,3,11a-四氢- $6 H$-色烯 并 [3,2-e]味唑并 $[1,2-a]$ 吡啶-6-酮(3bi): 黄色固体, m.p. $237 \sim 238{ }^{\circ} \mathrm{C} ;{ }^{1} \mathrm{H}$ NMR (500 MHz, DMSO- $\left.d_{6}\right) \delta: 7.94(\mathrm{~s}$, $1 \mathrm{H}, \mathrm{CH}), 7.48 \sim 7.52(\mathrm{~m}, 2 \mathrm{H}, \mathrm{ArH}), 7.41 \sim 7.46(\mathrm{~m}, 1 \mathrm{H}$, ArH), $7.26 \sim 7.29(\mathrm{~m}, 1 \mathrm{H}, \operatorname{ArH}), 7.22(\mathrm{~d}, J=8.0 \mathrm{~Hz}, 1 \mathrm{H}$, ArH), $7.14 \sim 7.18$ (m, 2H, ArH), 6.43 (s, 1H, CH), 4.89 (d, $\left.J=16.5 \mathrm{~Hz}, 1 \mathrm{H}, \mathrm{CH}_{2}\right), 4.72\left(\mathrm{~d}, J=16.5 \mathrm{~Hz}, 1 \mathrm{H}, \mathrm{CH}_{2}\right)$, $4.13 \sim 4.17\left(\mathrm{~m}, 1 \mathrm{H}, \mathrm{CH}_{2}\right), 3.90 \sim 3.94\left(\mathrm{~m}, 2 \mathrm{H}, \mathrm{CH}_{2}\right)$, $3.81 \sim 3.85\left(\mathrm{~m}, 1 \mathrm{H}, \mathrm{CH}_{2}\right) ;{ }^{13} \mathrm{C}$ NMR $\left(125 \mathrm{MHz}, \mathrm{DMSO}-d_{6}\right)$ $\delta: 178.5,162.8\left(\mathrm{~d},{ }^{1} J_{\mathrm{C}-\mathrm{F}}=241.3 \mathrm{~Hz}\right), 157.8\left(\mathrm{~d},{ }^{1} J_{\mathrm{C}-\mathrm{F}}=\right.$ $238.8 \mathrm{~Hz}), 155.2,152.9,139.2\left(\mathrm{~d},{ }^{3} J_{\mathrm{C}-\mathrm{F}}=7.5 \mathrm{~Hz}\right), 131.1$, $130.9\left(\mathrm{~d},{ }^{3} J_{\mathrm{C}-\mathrm{F}}=8.8 \mathrm{~Hz}\right), 124.4\left(\mathrm{~d},{ }^{3} J_{\mathrm{C}-\mathrm{F}}=6.3 \mathrm{~Hz}\right), 123.9$, $123.7\left(\mathrm{~d},{ }^{2} J_{\mathrm{C}-\mathrm{F}}=23.8 \mathrm{~Hz}\right), 120.8\left(\mathrm{~d},{ }^{3} J_{\mathrm{C}-\mathrm{F}}=7.5 \mathrm{~Hz}\right), 114.9$, 
$114.7,112.3\left(\mathrm{~d},{ }^{2} J_{\mathrm{C}-\mathrm{F}}=22.5 \mathrm{~Hz}\right), 111.4,110.6,86.1,54.7$, 51.1, 45.6; IR (KBr) v: 3076, 1655, 1608, 1553, 1484, 1284, 1251, 1215, 949, $620 \mathrm{~cm}^{-1}$; HRMS (TOF ES ${ }^{+}$) calcd for $\mathrm{C}_{21} \mathrm{H}_{16} \mathrm{~F}_{2} \mathrm{~N}_{3} \mathrm{O}_{4}[\mathrm{M}+\mathrm{H}]^{+}$412.1103, found 412.1101.

3-(3,5-二氟芐基)-8-氟-4-硝基-1,2,3,11 $a$-四氢- $6 H$-色 烯并[3,2-e]咪唑并 $[1,2-a]$ 吡啶-6-酮(3bj): 黄色固体, m.p. 256 257 ${ }^{\circ} \mathrm{C} ;{ }^{1} \mathrm{H}$ NMR (500 MHz, DMSO- $d_{6}$ ) $\delta: 7.94$ (s, $1 \mathrm{H}, \mathrm{CH}), 7.49 \sim 7.53(\mathrm{~m}, 2 \mathrm{H}, \mathrm{ArH}), 7.16 \sim 7.17(\mathrm{~m}, 4 \mathrm{H}$, $\mathrm{ArH}), 6.44(\mathrm{~s}, 1 \mathrm{H}, \mathrm{CH}), 4.88\left(\mathrm{~d}, J=17.0 \mathrm{~Hz}, 1 \mathrm{H}, \mathrm{CH}_{2}\right)$, $4.68\left(\mathrm{~d}, J=16.5 \mathrm{~Hz}, 1 \mathrm{H}, \mathrm{CH}_{2}\right), 4.16 \sim 4.18\left(\mathrm{~m}, 1 \mathrm{H}, \mathrm{CH}_{2}\right)$, $3.91 \sim 3.95\left(\mathrm{~m}, 2 \mathrm{H}, \mathrm{CH}_{2}\right), 3.82 \sim 3.86\left(\mathrm{~m}, 1 \mathrm{H}, \mathrm{CH}_{2}\right) ;{ }^{13} \mathrm{C}$ NMR (125 MHz, DMSO- $\left.d_{6}\right) \delta: 178.5,162.9\left(\mathrm{dd},{ }^{1} J_{\mathrm{C}-\mathrm{F}}=\right.$ $\left.244.4 \mathrm{~Hz},{ }^{3} J_{\mathrm{C}-\mathrm{F}}=13.1 \mathrm{~Hz}\right), 162.9\left(\mathrm{~d},{ }^{1} J_{\mathrm{C}-\mathrm{F}}=245.0 \mathrm{~Hz}\right)$, $157.8\left(\mathrm{~d},{ }^{1} J_{\mathrm{C}-\mathrm{F}}=238.8 \mathrm{~Hz}\right), 155.5,152.9,141.3,131.1$, $124.4,123.7\left(\mathrm{~d},{ }^{2} J_{\mathrm{C}-\mathrm{F}}=25.0 \mathrm{~Hz}\right), 120.8\left(\mathrm{~d},{ }^{3} J_{\mathrm{C}-\mathrm{F}}=7.5 \mathrm{~Hz}\right)$, $112.3\left(\mathrm{~d},{ }^{2} J_{\mathrm{C}-\mathrm{F}}=22.5 \mathrm{~Hz}\right), 111.5,111.0\left(\mathrm{~d},{ }^{2} J_{\mathrm{C}-\mathrm{F}}=25.0 \mathrm{~Hz}\right)$, $110.5,103.3(\mathrm{~m}), 86.1,54.7,51.2,45.7 ;{ }^{19} \mathrm{~F}$ NMR (470 MHz, DMSO- $\left.d_{6}\right) \delta:-109.9,-119.8$; IR (KBr) v: 3080, $1657,1598,1554,1482,1299,1252,1217,1119,612$ $\mathrm{cm}^{-1}$; HRMS (TOF ES ${ }^{+}$) calcd for $\mathrm{C}_{21} \mathrm{H}_{15} \mathrm{~F}_{3} \mathrm{~N}_{3} \mathrm{O}_{4}[\mathrm{M}+$ $\mathrm{H}]^{+}$430.1009, found 430.1008 .

3-((6-氯吡啶-3-基)甲基)-8-氟-4-硝基-1,2,3,11a-四 氢- $6 H$-色烯并 $[3,2-e]$ 咪唑并 $[1,2-a]$ 吡啶-6-酮(3bk): 黄色 固体, m.p. $218 \sim 219{ }^{\circ} \mathrm{C} ;{ }^{1} \mathrm{H}$ NMR (500 MHz, DMSO- $d_{6}$ ) $\delta: 8.46$ (s, $1 \mathrm{H}, \mathrm{CH}), 7.90 \sim 7.93(\mathrm{~m}, 2 \mathrm{H}, \mathrm{ArH}), 7.49 \sim 7.56$ (m, 3H, ArH), 7.15 7.18 (m, 1H, ArH), $6.43(\mathrm{~s}, 1 \mathrm{H}, \mathrm{CH})$, $4.90\left(\mathrm{~d}, J=16.0 \mathrm{~Hz}, 1 \mathrm{H}, \mathrm{CH}_{2}\right), 4.73(\mathrm{~d}, J=16.5 \mathrm{~Hz}, 1 \mathrm{H}$, $\left.\mathrm{CH}_{2}\right), 4.14 \sim 4.17\left(\mathrm{~m}, 1 \mathrm{H}, \mathrm{CH}_{2}\right), 3.92 \sim 3.96\left(\mathrm{~m}, 2 \mathrm{H}, \mathrm{CH}_{2}\right)$, $3.81 \sim 3.85\left(\mathrm{~m}, 1 \mathrm{H}, \mathrm{CH}_{2}\right) ;{ }^{13} \mathrm{C}$ NMR (125 MHz, DMSO- $d_{6}$ ) $\delta: 178.5,157.8\left(\mathrm{~d},{ }^{1} J_{\mathrm{C}-\mathrm{F}}=240.0 \mathrm{~Hz}\right), 155.4,152.9,149.9$, $149.4,139.4,131.7,131.0,124.5,124.4\left(\mathrm{~d},{ }^{3} J_{\mathrm{C}-\mathrm{F}}=6.3 \mathrm{~Hz}\right)$, $123.7\left(\mathrm{~d},{ }^{2} J_{\mathrm{C}-\mathrm{F}}=23.8 \mathrm{~Hz}\right), 120.8\left(\mathrm{~d},{ }^{3} J_{\mathrm{C}-\mathrm{F}}=7.5 \mathrm{~Hz}\right), 112.3$ $\left(\mathrm{d},{ }^{2} J_{\mathrm{C}-\mathrm{F}}=23.8 \mathrm{~Hz}\right), 111.6,110.5,86.0,52.6,51.1,45.7$; IR (KBr) $v: 3074,1661,1575,1560,1481,1315,1257,1211$, $616 \mathrm{~cm}^{-1}$; HRMS (TOF ES ${ }^{+}$) calcd for $\mathrm{C}_{20} \mathrm{H}_{15} \mathrm{ClFN}_{4} \mathrm{O}_{4}$ $[\mathrm{M}+\mathrm{H}]^{+}$429.0760, found 429.0762 .

3-((2-氯噻唑-5-基)甲基)-8-氟-4-硝基-1,2,3,11a-四 氢- $6 H$-色烯并 $[3,2-e]$ 咪唑并 $[1,2 a]$ 吡啶-6-酮(3bl): 黄色 固体, m.p. $230 \sim 231{ }^{\circ} \mathrm{C} ;{ }^{1} \mathrm{H}$ NMR (500 MHz, DMSO- $d_{6}$ ) $\delta: 7.94(\mathrm{~s}, 1 \mathrm{H}, \mathrm{CH}), 7.79(\mathrm{~s}, 1 \mathrm{H}, \mathrm{ArH}), 7.49 \sim 7.52(\mathrm{~m}, 2 \mathrm{H}$, ArH), $7.15 \sim 7.17(\mathrm{~m}, 1 \mathrm{H}, \mathrm{ArH}), 6.42(\mathrm{~s}, 1 \mathrm{H}, \mathrm{CH}), 4.99(\mathrm{~d}$, $\left.J=15.5 \mathrm{~Hz}, 1 \mathrm{H}, \mathrm{CH}_{2}\right), 4.84\left(\mathrm{~d}, J=16.0 \mathrm{~Hz}, 1 \mathrm{H}, \mathrm{CH}_{2}\right)$, $4.14 \sim 4.15\left(\mathrm{~m}, 1 \mathrm{H}, \mathrm{CH}_{2}\right), 3.89 \sim 3.96\left(\mathrm{~m}, 2 \mathrm{H}, \mathrm{CH}_{2}\right)$, $3.78 \sim 3.80\left(\mathrm{~m}, 1 \mathrm{H}, \mathrm{CH}_{2}\right) ;{ }^{13} \mathrm{C}$ NMR $\left(125 \mathrm{MHz}\right.$, DMSO- $\left.d_{6}\right)$ $\delta: 178.5,157.8\left(\mathrm{~d},{ }^{1} J_{\mathrm{C}-\mathrm{F}}=238.8 \mathrm{~Hz}\right), 154.7,152.8,152.3$, $142.3,135.6,130.7,124.3\left(\mathrm{~d},{ }^{3} J_{\mathrm{C}-\mathrm{F}}=7.5 \mathrm{~Hz}\right), 123.8(\mathrm{~d}$, $\left.{ }^{2} J_{\mathrm{C}-\mathrm{F}}=25.0 \mathrm{~Hz}\right), 120.8\left(\mathrm{~d},{ }^{3} J_{\mathrm{C}-\mathrm{F}}=7.5 \mathrm{~Hz}\right), 112.3\left(\mathrm{~d},{ }^{2} J_{\mathrm{C}-\mathrm{F}}=\right.$ $23.8 \mathrm{~Hz}), 112.1,110.5,85.8,50.4,48.6$, 45.5; IR (KBr) $v$ : $3067,1663,1568,1482,1258,1213,1049,615 \mathrm{~cm}^{-1}$; HRMS (TOF ES ${ }^{+}$) calcd for $\mathrm{C}_{18} \mathrm{H}_{13} \mathrm{ClFN}_{4} \mathrm{O}_{4} \mathrm{~S}[\mathrm{M}+\mathrm{H}]^{+}$ 435.0325, found 435.0327 .

8-氯-4-硝基-3-(4-(三氟甲基)苄基)-1,2,3,11 $a$-四氢$6 H$-苯并吡喃并 $[3,2-e]$ 咪唑并 $[1,2-a]$ 吡啶-6-酮(3cb): 黄 色固体, m.p. 243 244 ${ }^{\circ} \mathrm{C}$; ${ }^{1} \mathrm{H}$ NMR (500 MHz, DMSO$\left.d_{6}\right) \delta: 7.94(\mathrm{~s}, 1 \mathrm{H}, \mathrm{CH}), 7.75 \sim 7.77(\mathrm{~m}, 3 \mathrm{H}, \mathrm{ArH}), 7.62 \sim$ $7.65(\mathrm{~m}, 3 \mathrm{H}, \mathrm{ArH}), 7.16(\mathrm{~d}, J=8.5 \mathrm{~Hz}, 1 \mathrm{H}, \mathrm{ArH}), 6.48(\mathrm{~s}$, $1 \mathrm{H}, \mathrm{CH}), 4.97$ (d, $\left.J=16.5 \mathrm{~Hz}, 1 \mathrm{H}, \mathrm{CH}_{2}\right), 4.81$ (d, $J=16.5$ $\left.\mathrm{Hz}, 1 \mathrm{H}, \mathrm{CH}_{2}\right), 4.17 \sim 4.18\left(\mathrm{~m}, 1 \mathrm{H}, \mathrm{CH}_{2}\right), 3.92 \sim 3.95(\mathrm{~m}$, $\left.2 \mathrm{H}, \mathrm{CH}_{2}\right), 3.83 \sim 3.87\left(\mathrm{~m}, 1 \mathrm{H}, \mathrm{CH}_{2}\right) ;{ }^{13} \mathrm{C} \mathrm{NMR}(125 \mathrm{MHz}$, DMSO- $\left.d_{6}\right) \delta: 178.5,155.3,155.2,141.3,136.0,131.3$, $128.5\left(\mathrm{~d},{ }^{3} J_{\mathrm{C}-\mathrm{F}}=31.3 \mathrm{~Hz}\right), 128.5,127.3,125.8(\mathrm{~m})$, $123.7 \sim 125.8(\mathrm{~m}), 124.7,121.0,111.2,110.6,86.2,55.0$, 51.2, 45.7; IR (KBr) v: 1655, 1614, 1557, 1478, 1327, 1264, 1121, 1066, $615 \mathrm{~cm}^{-1}$; HRMS (TOF ES ${ }^{+}$) calcd for $\mathrm{C}_{22} \mathrm{H}_{16} \mathrm{ClF}_{3} \mathrm{~N}_{3} \mathrm{O}_{4}[\mathrm{M}+\mathrm{H}]^{+}$478.0776, found 478.0776.

8-氯-3-(4-氟苄基)-4-硝基-1,2,3,11a-四氢- $6 H$-色烯 并 [3,2-e ]咪唑并 $[1,2-a]$ 吡啶-6-酮(3cd): 黄色固体, m.p. $242 \sim 243{ }^{\circ} \mathrm{C} ;{ }^{1} \mathrm{H}$ NMR $\left(500 \mathrm{MHz}, \mathrm{DMSO}-d_{6}\right) \delta: 7.93$ (s, $1 \mathrm{H}, \mathrm{CH}), 7.75(\mathrm{~d}, J=2.5 \mathrm{~Hz}, 1 \mathrm{H}, \mathrm{ArH}), 7.64 \sim 7.67(\mathrm{~m}$, $1 \mathrm{H}, \mathrm{ArH}), 7.43 \sim 7.46(\mathrm{~m}, 2 \mathrm{H}, \mathrm{ArH}), 7.20 \sim 7.24(\mathrm{~m}, 2 \mathrm{H}$, ArH), 7.14 (d, $J=9.0 \mathrm{~Hz}, 1 \mathrm{H}, \mathrm{ArH}), 6.45(\mathrm{~s}, 1 \mathrm{H}, \mathrm{CH}), 4.85$ (d, $\left.J=15.0 \mathrm{~Hz}, 1 \mathrm{H}, \mathrm{CH}_{2}\right), 4.72\left(\mathrm{~d}, J=15.0 \mathrm{~Hz}, 1 \mathrm{H}, \mathrm{CH}_{2}\right)$, $4.13 \sim 4.15\left(\mathrm{~m}, 1 \mathrm{H}, \mathrm{CH}_{2}\right), 3.87 \sim 3.91\left(\mathrm{~m}, 2 \mathrm{H}, \mathrm{CH}_{2}\right), 3.81$ $\left(\mathrm{m}, 1 \mathrm{H}, \mathrm{CH}_{2}\right) ;{ }^{13} \mathrm{C}$ NMR $\left(125 \mathrm{MHz}\right.$, DMSO- $\left.d_{6}\right) \delta: 178.2$, $162.1\left(\mathrm{~d},{ }^{1} J_{\mathrm{C}-\mathrm{F}}=242.5 \mathrm{~Hz}\right), 155.2,154.9,135.7,132.1(\mathrm{~d}$, $\left.{ }^{4} J_{\mathrm{C}-\mathrm{F}}=2.5 \mathrm{~Hz}\right), 131.3,130.2\left(\mathrm{~d},{ }^{3} J_{\mathrm{C}-\mathrm{F}}=7.5 \mathrm{~Hz}\right), 127.2$, $126.3,124.7,121.0,115.8\left(\mathrm{~d},{ }^{2} J_{\mathrm{C}-\mathrm{F}}=21.3 \mathrm{~Hz}\right), 111.1$, 110.7, 86.2, 54.3, 50.9, 45.6; IR (KBr) v: 3072, 1655, $1615,1562,1479,1362,1270,1213,619 \mathrm{~cm}^{-1}$; HRMS (TOF ES ${ }^{+}$) calcd for $\mathrm{C}_{21} \mathrm{H}_{16} \mathrm{ClFN}_{3} \mathrm{O}_{4}[\mathrm{M}+\mathrm{H}]^{+} 428.0808$, found 428.0810 .

8-氯-3-(4-甲基苄基)-4-硝基-1,2,3,11a-四氢- $6 \mathrm{H}$-色 烯并[3,2-e]咪唑并 $[1,2-a]$ 吡啶-6-酩(3cg): 黄色固体, m.p. $233 \sim 234{ }^{\circ} \mathrm{C} ;{ }^{1} \mathrm{H}$ NMR (500 MHz, DMSO- $d_{6}$ ) $\delta: 7.93$ (s, $1 \mathrm{H}, \mathrm{CH}), 7.75(\mathrm{~d}, J=2.5 \mathrm{~Hz}, 1 \mathrm{H}, \mathrm{ArH}), 7.64 \sim 7.67(\mathrm{~m}$, $1 \mathrm{H}, \operatorname{ArH}), 7.14 \sim 7.27(\mathrm{~m}, 5 \mathrm{H}, \mathrm{ArH}), 6.45(\mathrm{~s}, 1 \mathrm{H}, \mathrm{CH})$, $4.73 \sim 4.80\left(\mathrm{~m}, 2 \mathrm{H}, \mathrm{CH}_{2}\right), 4.10 \sim 4.14\left(\mathrm{~m}, 1 \mathrm{H}, \mathrm{CH}_{2}\right)$, $3.88 \sim 3.91\left(\mathrm{~m}, 2 \mathrm{H}, \mathrm{CH}_{2}\right), 3.78 \sim 3.82\left(\mathrm{~m}, 1 \mathrm{H}, \mathrm{CH}_{2}\right), 2.31$ $\left(\mathrm{s}, 3 \mathrm{H}, \mathrm{CH}_{3}\right) ;{ }^{13} \mathrm{C}$ NMR $\left(125 \mathrm{MHz}, \mathrm{DMSO}-d_{6}\right) \delta: 178.1$, $155.2,154.8,137.4,136.0,132.9,129.6,128.2,127.2$, 
$126.3,124.7,121.0,111.1,86.3,54.5,50.9,45.5,21.2$; IR (KBr) v: 3071, 1654, 1614, 1562, 1480, 1376, 1270, 1215, $619 \mathrm{~cm}^{-1}$; HRMS (TOF ES ${ }^{+}$) calcd for $\mathrm{C}_{22} \mathrm{H}_{19} \mathrm{ClN}_{3} \mathrm{O}_{4}[\mathrm{M}$ $+\mathrm{H}]^{+}$424.1059, found 424.1057 .

8-氯-3-(4-甲氧基苠基)-4-硝基-1,2,3,11 $a$-四氢- $6 H$ 色烯并 $[3,2-e]$ 咪唑并 $[1,2-a]$ 吡啶-6-酮(3ch): 黄色固体, m.p. $218 \sim 219{ }^{\circ} \mathrm{C} ;{ }^{1} \mathrm{H}$ NMR (500 MHz, DMSO- $\left.d_{6}\right) \delta$ : $7.93(\mathrm{~s}, 1 \mathrm{H}, \mathrm{CH}), 7.74(\mathrm{~d}, J=3.0 \mathrm{~Hz}, 1 \mathrm{H}, \mathrm{ArH}), 7.63 \sim$ 7.66 (m, 1H, ArH), 7.31 (d, $J=8.5 \mathrm{~Hz}, 2 \mathrm{H}, \mathrm{ArH}), 7.14$ (d, $J=8.5 \mathrm{~Hz}, 1 \mathrm{H}, \mathrm{ArH}), 6.95$ (d, $J=8.5 \mathrm{~Hz}, 2 \mathrm{H}, \mathrm{ArH}), 6.44$ (s, 1H, CH), 4.78 (d, $\left.J=14.0 \mathrm{~Hz}, 1 \mathrm{H}, \mathrm{CH}_{2}\right), 4.69$ (d, $J=$ $\left.14.5 \mathrm{~Hz}, 1 \mathrm{H}, \mathrm{CH}_{2}\right), 4.11 \sim 4.12\left(\mathrm{~m}, 1 \mathrm{H}, \mathrm{CH}_{2}\right), 3.86 \sim 3.89$ (m, $\left.2 \mathrm{H}, \mathrm{CH}_{2}\right), 3.74 \sim 3.78\left(\mathrm{~m}, 1 \mathrm{H}, \mathrm{CH}_{2}\right), 3.76\left(\mathrm{~s}, 3 \mathrm{H}, \mathrm{CH}_{3}\right)$; ${ }^{13} \mathrm{C}$ NMR (125 MHz, DMSO- $\left.d_{6}\right) \delta: 178.2,159.4,155.2$, $154.6,135.9,131.4,129.7,127.6,127.2,126.3,124.7$, $121.0,114.5,111.0,110.7,86.2,55.6,54.1,50.7,45.5$; IR (KBr) v: 2932, 1651, 1610, 1557, 1509, 1279, 1245, 1221, $1119,620 \mathrm{~cm}^{-1}$; HRMS (TOF $\mathrm{ES}^{+}$) calcd for $\mathrm{C}_{22} \mathrm{H}_{19} \mathrm{Cl}-$ $\mathrm{N}_{3} \mathrm{O}_{5}[\mathrm{M}+\mathrm{H}]^{+}$440.1008, found 440.1008.

4-((8-甲基-4-硝基-6-氧代-1,2-二氢- $6 H$-色烯并 [3,2-e]咪唑并 $[1,2-a]$ 吡啶-3(11aH)-基)甲基)芐腈(3dc): 黄色固体, m.p. $290 \sim 291{ }^{\circ} \mathrm{C}$; ${ }^{1} \mathrm{H}$ NMR $(600 \mathrm{MHz}$, DMSO-d $d_{6} \delta:$ 7.90(s, 1H, CH), 7.87 (d, $J=7.9 \mathrm{~Hz}, 1 \mathrm{H}$, $\operatorname{ArH}), 7.59 \sim 7.63(\mathrm{~m}, 3 \mathrm{H}, \operatorname{ArH}), 7.45(\mathrm{~d}, J=8.2 \mathrm{~Hz}, 1 \mathrm{H}$, ArH), 7.02 (d, J=8.4 Hz, 1H, ArH), 6.40 (s, 1H, CH), 4.96 (d, $\left.J=17.0 \mathrm{~Hz}, 1 \mathrm{H}, \mathrm{CH}_{2}\right), 4.78\left(\mathrm{~d}, J=16.9 \mathrm{~Hz}, 1 \mathrm{H}, \mathrm{CH}_{2}\right.$ ), $4.16 \sim 4.20\left(\mathrm{~m}, 1 \mathrm{H}, \mathrm{CH}_{2}\right), 3.91 \sim 3.94\left(\mathrm{~m}, 2 \mathrm{H}, \mathrm{CH}_{2}\right), 3.81 \sim$ $3.86\left(\mathrm{~m}, 1 \mathrm{H}, \mathrm{CH}_{2}\right), 2.32\left(\mathrm{~s}, 3 \mathrm{H}, \mathrm{CH}_{3}\right) ;{ }^{13} \mathrm{C} \mathrm{NMR}(150$ MHz, DMSO- $\left.d_{6}\right) \delta: 179.4,155.5,154.6,142.5,137.3$, $132.8,132.4,130.2,128.6,127.0,123.2,119.2,118.5$, $112.6,110.6,110.3,85.7,55.2,51.3,45.6,20.6$; IR (KBr) $v: 2223,1658,1622,1554,1507,1485,1307,1288,1218$, $623 \mathrm{~cm}^{-1}$; HRMS (TOF ES ${ }^{+}$) calcd for $\mathrm{C}_{23} \mathrm{H}_{19} \mathrm{~N}_{4} \mathrm{O}_{4}[\mathrm{M}+$ $\mathrm{H}^{+}$415.1401, found 415.1398 .

(4-甲氧基苄基)-8-甲基-4-硝基-1,2,3,11a-四氢- $6 H$ 色烯并 [3,2-e]咪唑并 $[1,2-a]$ 吡啶-6-酮(3dh): 黄色固体, m.p. $207 \sim 208{ }^{\circ} \mathrm{C}$; ${ }^{1} \mathrm{H}$ NMR (600 MHz, DMSO- $\left.d_{6}\right) \delta$ : 7.91 (s, 1H, CH), 7.62 (s, 1H, CH), 7.43 (d, $J=8.3 \mathrm{~Hz}, 1 \mathrm{H}$, $\operatorname{ArH}), 7.31(\mathrm{~d}, J=7.9 \mathrm{~Hz}, 2 \mathrm{H}, \operatorname{ArH}), 6.99$ (d, $J=8.3 \mathrm{~Hz}$, $1 \mathrm{H}, \operatorname{ArH}), 6.95$ (d, $J=8.22 \mathrm{~Hz}, 2 \mathrm{H}, \mathrm{ArH}), 6.36$ (s, 1H, $\mathrm{CH}), 4.80\left(\mathrm{~d}, J=15.2 \mathrm{~Hz}, 1 \mathrm{H}, \mathrm{CH}_{2}\right), 4.69$ (d, $J=15.2 \mathrm{~Hz}$, $\left.1 \mathrm{H}, \mathrm{CH}_{2}\right), 4.09 \sim 4.13\left(\mathrm{~m}, 1 \mathrm{H}, \mathrm{CH}_{2}\right), 3.85 \sim 3.88(\mathrm{~m}, 2 \mathrm{H}$, $\left.\mathrm{CH}_{2}\right), 3.74 \sim 3.78\left(\mathrm{~m}, 1 \mathrm{H}, \mathrm{CH}_{2}\right), 3.76\left(\mathrm{~s}, 3 \mathrm{H}, \mathrm{CH}_{3}\right), 2.31(\mathrm{~s}$, $\left.3 \mathrm{H}, \mathrm{CH}_{3}\right) ;{ }^{13} \mathrm{C} \mathrm{NMR}\left(150 \mathrm{MHz}, \mathrm{DMSO}-d_{6}\right) \delta: 179.3$, $159.3,154.8,154.6,137.3,132.3,130.4,129.7,127.0$,
$123.2,118.4,114.5,112.4,110.4,85.8,55.6,54.1,50.6$, 45.4, 20.6; IR (KBr) v: 1655, 1617, 1554, 1504, 1311, $1275,1212,1195,623 \mathrm{~cm}^{-1}$; HRMS (TOF ES ${ }^{+}$) calcd for $\mathrm{C}_{23} \mathrm{H}_{22} \mathrm{~N}_{3} \mathrm{O}_{5}[\mathrm{M}+\mathrm{H}]^{+}$420.1554, found 420.1557.

3-((6-氯吡啶-3-基)甲基)-8-甲基-4-硝基-1,2,3,11a四氢- $6 H$-色烯并 $[3,2-e]$ 咪唑并 $[1,2-a$ ]吡啶-6-酮(3dk): 黄 色固体, m.p. $248 \sim 249{ }^{\circ} \mathrm{C} ;{ }^{1} \mathrm{H}$ NMR $(600 \mathrm{MHz}$, DMSO- $\left.d_{6}\right) \delta: 8.46(\mathrm{~s}, 1 \mathrm{H}, \mathrm{CH}), 7.91(\mathrm{~s}, 2 \mathrm{H}, \mathrm{ArH}), 7.63(\mathrm{~s}$, $1 \mathrm{H}, \mathrm{ArH}), 7.57$ (d, $J=7.5 \mathrm{~Hz}, 1 \mathrm{H}, \mathrm{ArH}), 7.45$ (d, $J=7.0$ $\mathrm{Hz}, 1 \mathrm{H}, \mathrm{ArH}), 7.01$ (d, J=7.5 Hz, 1H, ArH), 6.39 (s, 1H, $\mathrm{CH}), 4.90\left(\mathrm{~d}, J=16.3 \mathrm{~Hz}, 1 \mathrm{H}, \mathrm{CH}_{2}\right), 4.72(\mathrm{~d}, J=16.2 \mathrm{~Hz}$, $\left.1 \mathrm{H}, \mathrm{CH}_{2}\right), 4.16 \sim 4.17\left(\mathrm{~m}, 1 \mathrm{H}, \mathrm{CH}_{2}\right), 3.93\left(\mathrm{~s}, 2 \mathrm{H}, \mathrm{CH}_{2}\right)$, $3.81 \sim 4.83\left(\mathrm{~m}, 1 \mathrm{H}, \mathrm{CH}_{2}\right), 2.32\left(\mathrm{~s}, 3 \mathrm{H}, \mathrm{CH}_{3}\right) ;{ }^{13} \mathrm{C} \mathrm{NMR}$ $\left(150 \mathrm{MHz}, \mathrm{DMSO}-d_{6}\right) \delta: 179.4,155.5,154.6,149.8,149.5$, $139.5,137.3,132.4,131.8,130.2,127.0,124.5,123.2$, $118.5,112.7,110.3,85.8,52.6,51.1,45.6,20.5$; IR (KBr) $v: 1661,1624,1555,1506,1452,1290,1216,623 \mathrm{~cm}^{-1}$; HRMS (TOF ES ${ }^{+}$) calcd for $\mathrm{C}_{21} \mathrm{H}_{18} \mathrm{ClN}_{4} \mathrm{O}_{4}[\mathrm{M}+\mathrm{H}]^{+}$ 425.1008, found 425.1008 .

辅助材料(Supporting Information) 化合物的 ${ }^{1} \mathrm{H}$ NMR 和 ${ }^{13} \mathrm{C}$ NMR 谱图. 这些材料可以免费从本刊网站(http:// sioc-journal.cn/)上下载.

\section{Referenes}

[1] Hunt, A. J.; Sin, E. H. K.; Marriott, R.; Clark, J. H. CHemSusChem 2010, 3, 306.

[2] Agana, B. A.; Reeve, D.; Orbell, J. D. J Environ. Manage. 2013, $114,445$.

[3] Marr, P. C.; Marr, A. C. Green Chem. 2016, 18, 105.

[4] Marteel-Parrish, A. E. J. Chem. Educ. 2014, 91, 1084.

[5] Trost, B. M. Science 1991, 254, 1471.

[6] Misono, M. Yuki Gosei Kagaku Kyokaishi 2003, 61, 406.

[7] Hartman, G. J.; Jin, Q. Z.; Collins, G. J.; Lee, K. N.; Ho, C. T.; Chang, S. S. J. Agric. Food Chem. 1983, 31, 1030.

[8] Ren, T.; Liu, W.; Xue, Q.; Wang, H. Lubr. Sci. 1993, 5, 205.

[9] Ueda, Y.; Connolly, T. P.; Kadow, J. F.; Meanwell, N. A.; Wang, T.; Chen, C.-P. H.; Yeung, K.-S.; Zhang, Z.; Leahy, D. K.; Pack, S. K.; Soundararajan, N.; Sirard, P.; Levesque, K.; Thoraval, D. US 20050209246, 2005 [Chem. Abstr. 2005, 143, 306343].

[10] Garuti, L.; Roberti, M.; Pizzirani, D. Mini-Rev. Med. Chem. 2007, $7,481$.

[11] Schultz, C.; Link, A.; Leost, M.; Zaharevitz, D. W.; Gussio, R.; Sausville, E. A.; Meijer, L.; Kunick, C. J. Med. Chem. 1999, 42, 2909.

[12] Pluta, K.; Morak-Mlodawska, B.; Jelen, M. Eur. J. Med. Chem. 2011, 46, 3179.

[13] Sujatha, K.; Shanmugam, P.; Perumal, P. T.; Muralidharan, D.; Rajendran, M. Bioorg. Med. Chem. Lett. 2006, 16, 4893.

[14] Helal, C. J.; Kang, Z.; Hou, X.; Pandit, J.; Chappie, T. A.; Humphrey, J. M.; Marr, E. S.; Fennell, K. F.; Chenard, L. K.; Fox, C.; Schmidt, C. J.; Williams, R. D.; Chapin, D. S.; Siuciak, J.; Lebel, L.; Menniti, F.; Cianfrogna, J.; Fonseca, K. R.; Nelson, F. R.; O'Connor, R.; MacDougall, M.; McDowell, L.; Liras, S. J. Med. Chem. 2011, 54, 4536.

[15] Kim, I. Y.; Kim, S. H. KR 2016006050, 2016 [Chem. Abstr. 2016, 
164, 225869].

[16] Lhassani, M.; Chavignon, O.; Chezal, J.-M.; Teulade, J.-C.; Chapat, J.-P.; Snoeck, R.; Andrei, G.; Balzarini, J.; De Clercq, E.; Gueiffier, A. Eur. J. Med. Chem. 1999, 34, 271.

[17] Alcarazo, M.; Roseblade, S. J.; Cowley, A. R.; Fernandez, R.; Brown, J. M.; Lassaletta, J. M. J. Am. Chem. Soc. 2005, 127, 3290.

[18] Mizushige, K.; Ueda, T.; Yukiiri, K.; Suzuki, H. Cardiovasc. Drug Rev. 2002, 20, 163.

[19] Ankley, G. T.; Kahl, M. D.; Jensen, K. M.; Hornung, M. W.; Korte, J. J.; Makynen, E. A.; Leino, R. L. Toxicol. Sci. 2002, 67, 121.

[20] Veber, D. F.; Johnson, S. R.; Cheng, H.-Y.; Smith, B. R.; Ward, K. W.; Kopple, K. D. J. Med. Chem. 2002, 45, 2615.

[21] Bai, D.; Lummis, S. C. R.; Leicht, W.; Breer, H.; Sattelle, D. B. Pestic. Sci. 1991, 33, 197.

[22] Feng, X.-G.; Liu, X.-W.; Han, Z.-L.; Guan, L.-T.; Xu, L.-Z. J. Qingdao Univ. Sci. Technol., Nat. Sci. Ed. 2012, 33, 381.

[23] Li, J.; Huang, T.; Li, L.; Ding, T.; Zhu, H.; Yang, B.; Ye, Q.; Gan, J. J. Agric. Food Chem. 2016, 64, 8109.

[24] Tomizawa, M.; Casida, J. E. Annu. Rev. Pharmacol. Toxicol. 2005, 45, 247.

[25] Bao, H.; Shao, X.; Zhang, Y.; Deng, Y.; Xu, X.; Liu, Z.; Li, Z. J. Agric. Food Chem. 2016, 64, 5148.

[26] Lu, S.; Zhuang, Y.; Wu, N.; Feng, Y.; Cheng, J.; Li, Z.; Chen, J.; Yuan, J.; Xu, X. J. Agric. Food Chem. 2013, 61, 10858

[27] Shao, X.; Xu, Z.; Zhao, X.; Xu, X.; Tao, L.; Li, Z.; Qian, X. J. Agric. Food Chem. 2010, 58, 2690.

[28] (a) Huang, Z.; Wang, M. Heterocycles 1994, 37, 1233.

(b) Kong, L.; Yang, R.; Du, X.; Yan, S.; Lin, J. Chin. J. Org. Chem. 2016, 36, 2437 (in Chinese).

(孔令斌, 杨瑞霞, 杜璇璇, 严胜骄, 林军, 有机化学, 2016, 36, 2437.) (c) Peng, M.; Yang, R.; Liu, X.; Yan, S.; Lin, J. Chin. J. Org. Chem. 2015, 35, 1754 (in Chinese).

(彭美阳，杨瑞霞，刘昔敏，严胜骄，林军，有机化学，2015，35, 1754.)

[29] (a) Chen, X.-B.; Liu, Z.-C.; Lin, X.-R.; Huang, R.; Yan, S.-J.; Lin, J. ACS Sustainable Chem. Eng. 2014, 2, 2391.

(b) Luo, D.; Cui, S.; Hu, X.; Yan, S.; Lin, J. Chin. J. Org. Chem. 2017, 37, 166 (in Chinese)

(罗大云，崔时胜，胡兴梅，林军，严胜骄，有机化学，2017，37, 166.)

[30] Yu, F.-C.; Huang, R.; Ni, H.; Fan, J.; Yan, S.-J.; Lin, J. Green Chem. 2013, 15, 453

[31] Chen, X.-B.; Liu, Z.-C.; Yang, L.-F.; Yan, S.-J.; Lin, J. ACS Sustainable Chem. Eng. 2014, 2, 1155.

[32] Xiao, X.; Wang, X.; Gui, X.; Chen, L.; Huang, B. Chem. Biodiversity 2016, 11, 1427.

[33] Mir, S. A. Int. J. PharmTech Res. 2016, 9, 70.

[34] (a) Ding, Z.-W.; Tan, Q.-T.; Liu, B.-X.; Xu, K.; Xu, B. Acta Chim. Sinica 2015, 73, 1302 (in Chinese).

(丁正伟，谭启涛，刘秉新，张可，许斌，化学学报，2015，73, 1302.)

(b) Zhao, J.-B.; Zhang, Q. Acta Chim. Sinica 2015, 73, 1235 (in Chinese). (赵金钵, 张前, 化学学报, 2015, 73, 1235.)

[35] Alizadeh, A.; Bayat, F.; Zhu, Z. Res. Chem. Intermed. 2016, 42, 5927.

[36] Yaqub, M.; Perveen, R.; Shafiq, Z.; Pervez, H.; Tahir, M. N. Synlett 2012, 23, 1755 .

[37] Nishiwaki, H.; Nakagawa, Y.; Takeda, D. Y.; Okazawa, A.; Akamatsu, M.; Miyagawa, H.; Ueno, T.; Nishimura, K. Pest Manage. Sci. 2000, 56, 875. 